\title{
ECONOMY OF FUEL IN ELECTRIC GENERATING STATIONS.
}

Bt Mr. henry McLaren, Member, of Leeds.

All Public or Municipal Lighting Authorities are required to farnish "Yearly Returns" to the Board of Trade, giving their costs of generating current. From these returns "The Flectrical Times" has compiled a Table of Costs, which is kept up to date, and published weekly. The following remarks and figures are based on this Table, published on 1st January 1903.

Most of the Municipal Bodies end their financial year on 31st March; therefore the figures given in Tables 1 to 11 (pages 523536) mainly refer to the financial year ending. March 1902. It must be borne in mind that this was a period of exoeptionally dear coal.

The first point that attracts attention is the great difference in cost per unit generated in these stations. They are located in various parts of the country; some are condensing, others partly condensing, or non-condensing stations, the price paid for coll raries largely, and different systems of supply are used. All these considerations affeot the costs.

In the retarns for the Lighting Stations, the figures are based on units sold to consumers, and therefore include the large leakage or loss in the mains, which is debited to the Generating Plant. This may be as much as 20 per cent., and is a very important factor to be kept in mind when comparing purely Lighting Stations with Tramway or Railway Plants, that "meter" the current as it leaves the Station. 
Engineers would naturally expect that condensing stations would show a better fuel economy than non-condensing, both for tram and lighting purposes, but so far as lighting only is concerned, it is just the reverse; the average, both in fuel and works costs, is considerably in favour of non-condensing stations. Where trams are added to lighting, the condensing plants show a better economy than non-condensing, but in all cases the partly condensing station is the most economical both in fuel and works costs.

Works costs include fuel, oil, water, waste, stores, wages of workmen, repairs and maintenance. It is manifestly unfair to compare purely Lighting Stations, which have only a poor load factor, with stations that combine Lighting and Tramways, and generate large quantities of current all day long, and meter it as it leaves the station.

The author has therefore subdivided the etations given in the Table of Costs published by "The Electrical Times," and arranged them as follows :-

22 Metropolitay Stations,

Lighting only.

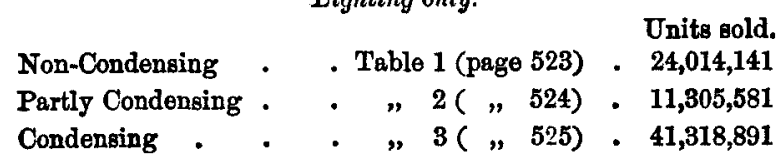

These stations are fairly comparable; they are all generating current for lighting purposes, and pay London prices for coal and labour. In comparing the costs of these three types, it is found that the non-condensing stations produce current at 18 per cent. and the partly condensing 21 per cent. less cost than the condensing stations. For fuel only, the saving in favour of non-condensing and partly condensing plants is about 14 per cent. and 17 per cent. respectively.

\section{Provinolal Stationg.}

Lighting only.

\begin{tabular}{|c|c|c|c|}
\hline \multicolumn{4}{|c|}{-50} \\
\hline Non-Condensing & & Table 4 (pages 526-7) & $\begin{array}{l}\text { Units sold } \\
\text { - } \quad 18,430,119\end{array}$ \\
\hline Partly Condensing & & $" 5$ (page 528) & $20,873,145$ \\
\hline Condensing & - & 6 (pages 529-531 & $35,933,215$ \\
\hline
\end{tabular}


These stations, as a rule, are small, and situated in various parts of the country; on the whole, they will have the advantage of cheaper coal and labour, as compared with the Metropolitan stations, and they show that their average cost of producing current is 18 per cent. less than the Metropolitan stations. Here again the non-condensing and partly condensing stations are more oconomical than the condensing, the saving in works costs being 14 per cent. and 16 per cent. respectively, and the saring in fuel $12 \frac{1}{2}$ per cent. and $20 \frac{1}{2}$ per cent.

32 Provinchal Stations.

Supplying Current both for Lighting and Tramways.

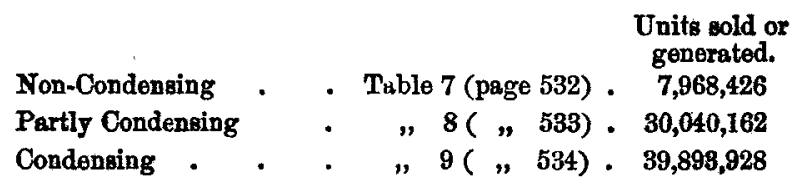

In these stations the condensing plants are found to be more economical than the non-condensing by about 9 per cent. in works costs, and 19 per cent. in cosl, but the partly condensing stations are still better than the condensing, showing an economy of 23 per cent. in works costs, and nearly 4 per cent. in fuel.

4 Provarolal Stations or Power-Hodgeg.

Generating Current for Electric Tramways.

All Condensing.

Total units generated . $\quad$. $\quad$ - 20,066,089

Table 10 (page 535) gives particulars of these Stations.

It is surprising to find that at least two of the Lighting Stations, Leeds (Table 6, page 530) and Edinburgh (Table 4, page 526), deliver current at the consumers' meters at 9 per cent. lower average cost than these four power-stations averaged at their switchboards; moreover Edinburgh is a non-condensing station.

Glasgow, the most economical of the four power-houses, with an annual output of over $10,000,000$ units and a tramway load-factor, delivers current at the switchboard at a cost of 0.6 penny per unit works costs; whereas Leeds Lighting Station, with only 3,000,000 output and a lighting load-factor, shows a works costs of 0.62 penny per unit, including the heavy loss in the mains. 
If the Leeds carrent was metered at the switchboard the works costs would read considerably lower than Glasgow, the most economical power-house in the country.

\author{
2 Metropolitan and 1 Provinoual Power-Hodgeg. \\ Generating Current for Electric Railways. \\ Total units generated $\quad . \quad$. 26,513,997 \\ Particulars of these Stations are given in Table 11 (page 536).
}

These are beaten in economy by the tramway power-houses, already mentioned, by over 20 per cent. in works costs, and $5 \frac{3}{4}$ per cent. in fuel costs. The extra for coal in the three railway power-houses may be accounted for by their geographical position, but the 20 per cent. excess in works costs appears unduly heavy.

The Diagram on the opposite page shows in graphic form the aversge costs of the various classes of stations or power-houses. The resul ts from any station given in the various Tables, may be compared with the average in its own, or any other class of station by means of this Table. Take Leeds Lighting Station, with fuel costs of 0.27 penny per unit, or works costs of 0.62 penny, it is found to be considerably lower than the average of the tramway power-stations, the lowest average on the diagram.

Taking coal at $10 \mathrm{lbs}$. for a penny, or 18s. 8d. per ton, then each decimal point of a penny on the diagram represents $1 \mathrm{lb}$. of coal. If the Metropolitan (Condensing) Lighting Stations, Table 3 (page 525), purchased their coal at 188. 8d. per ton, they would appear to be using on an average over $11 \frac{1}{2}$ lbs. of coal per unit sold, or 8.6 lbs. per electrical H.P., or say $7 \frac{1}{2}$ lbs. per I.H.P. per hour, taking a combined efficiency of 87 per cent. During the period under review these stations were no doubt paying more than $18 s$. $8 d$. per ton. This would modify the above figures, but it is impossible that coal could have been sufficiently dear to bring them within reasonable limits. An economical condensing engine and boiler should not require more than $1 \frac{1}{2}$ lbs, of good coal per I.H.P. per hour, or $2 \frac{1}{4}$ lbs. per unit generated. In everyday work, $2 \frac{1}{2}$ lbs. per unit at the switchboard may be considered very good economy.

From these figures it would appear that the Metropolitan Condensing Stations, Table 3 (page 525), after allowing 30 per cent. 
Diagram of Average Costs.

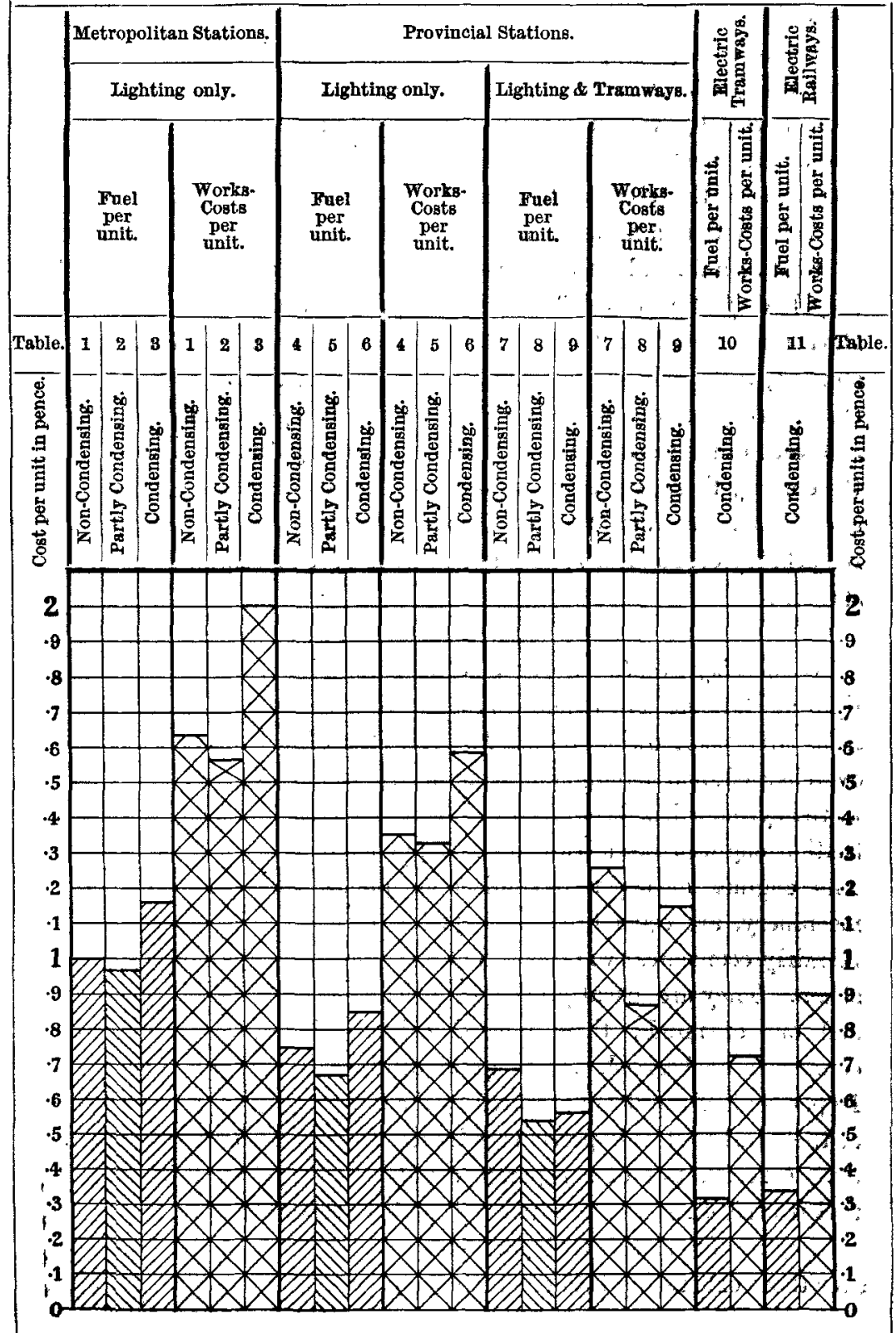

Works-Costs include Fuel, Oil, Waste, Water, Stores, Wages of Workmen, Repairs, and Maintenance. Downloaded from pme.sagepub.com at UNIV NEBRRASKA LIBRARIES on June 4, 2016 
for outside losses and banking fires, \&c., were using at least twice the amount of fuel that economical plants ought to require. They generated during the year over $41,000,000$ units, at a cost for fuel of over $£ 200,000$. A very large percentage of this amount could have been saved by the use of well-managed, economical generating plant. It must be remombered that most of these stations wero pionoers in the electric lighting industry of this country. Each extension was in a measure a large experiment. They have had to contend with difficulties too numerous to mention, and, like "Topsy," they have just "growed" to what they are. Could their engineers start afresh, with a "clean slate," there is no doubt that these stations, with their enormous output, would take their place amongst the most economical in the country. There is a good deal to be said in favour of the "clean slate." Taking the average station throughout the country there is great room for improvement; on the other hand, one finds a few stations that leave nothing to be desired; some remarkably economical results are recorded even in small provincial stations.

Condensing Plants.-Separate condensing plants are used in most lighting stations. The vacuum in themain engine low-pressure cylinder is usually poor, owing to the long lengths of exhaust pipe, often overcharged, and having numerous sharp bends; 11 inches vacuum in the cylinder has been recorded with 23 inches in the condenser. Station engineers as a rule do not pay sufficient attention to this. The engines of separate condensing plants, and other auxiliary machinery, are often fitted with piston valves. Such engines may be fairly economical when new, but soon develop a great appetite for steam. They are usually fixed in inaccessible places out of sight, and out of mind; their inflated exhaust passes direct to the condenser unobserved. It is quite common to find this type using 10 per cent. of the steam required by the main engines, when working at their full load, this percentage increasing rapidly as the load falls off. A recent test of a separate condensing plant revealed the fact that it used $4 \mathrm{lbs}$. of steam per unit generated by the main engines.

Engines fitted with their own condensers have a great advantage orer those exhausting into one main serving several engines; ususlly 
a saving of 5 to 10 per cent., due to the better vacuum, is effected. Add to this the 10 per cent. of steam used by the separate plant; the loss in the auxiliary steam main with its traps; the lose in starting up separate plants for short runs; the loss at light loads; the loss of vacuum through leaky atmospheric exhaust valves; and it is not so surprising that under these conditions, the economy due to condensing reaches the vanishing point, or even falls 13 per cent. behind well-equipped non-condensing stations, as shown by the Tables. The author's firm usually guaranteo $7 \frac{1}{2}$ per cent. less steam per kilowatt-hour if the engines are to be fitted with their own condensers, and drive their own pumps. The total cost of these engines is less than those fitted with separate condensers, and the steam required by the auxiliary machinery is altogether saved.

Partly-Oondensing Stations. - It is rather remarkable that the partly-condensing stations show the best average economy. Partly condensing is a very vague term; it may mean one engine in ten is non-condensing, or vice versâ. No doubt, most of them might be classed as condensing stations. Without more exact data it is impossible to come to any definite conclusion as to the reason for this good average economy.

Non-Condensing Stations.-In "The Engineer" of 15th May, 1903, the compound engines for the Tooting Electric Tramways are illustrated, and a few particulars are given as to guarantees, \&c. The lowest guarantee, when working condensing, is $13 \mathrm{f}$ lbs. of steam per I.H.P. per hour; the lowest when working non-condensing is 16 lbs. per I.H.P. per hour. If the latter figures cen be obtained, the money sunk in condensers, \&c., will only yiold a poor return, after the steam used by the condensing plant has been debited to the main engines. It is well to note that the figures here given are those guaranteed; the figures actually obtained have not been published.

About fifteen years ago, the author's firm devoted considerable attention to the manufacture of small compound non-condensing engines and boilers combined. Professor Barr and Mr. Druitt Halpin carefally tested one of the smallest sizes of these engines, with the following results, when usingian exhaust steam feed-heater:- 
Size of Cylinders :-5色 inches and 9 inches by 15 inches stroke, working at 24 I.H.P.

Steam per I.H.P.:-18. 8 Ibs. per hour.

Welsh coal per I.H.P. :-1.8 lbs. per hour.

Water evaporated per lb. of coal, from a temperature of $203^{\circ}$ F. :10.44 lbs. Boiler pressure, 140 lbs.

Mechanical efficiency of engine, 86 per cent.

The above results in fuel and steam may be considered very good for non-condensing compound engines even of much larger sizes.

The results obtained from the smallest size of triple-expansion condensing engines, made by the same firm, and tested by $\mathrm{Mr}$. Wilson Hartnell and Professor Goodman, were as follows :-

Sizes of Cylinders:-9 inches, $14 \frac{4}{4}$ inches, and $22 \frac{1}{2}$ inches by 24 inches stroke, working at 120 I.H.P.

Steam per I.H.P. per hour:-12.54 lbs.

Welsh coal per I.H.P. per hour : $-1 \cdot 25 \mathrm{lbs}$.

Water evaporated per $1 \mathrm{~b}$. of coal from a temperature of $110^{\circ} \mathrm{F} .:-$ $10 \cdot 11 \mathrm{lbs}$.

Water evaporated per $\mathrm{lb}$. of coal from and at $212^{\circ} \mathrm{F} .:-11 \cdot 34 \mathrm{lbs}$.

Boiler pressure:-157 lbs. per square inch.

Mechanical efficiency of engine, including the driving of all the pumps, and brake-wheel friction: $-93 \cdot 5$ per cent.

These triple engines were fitted with surface condensers, and drove their own air, circulating, and feed-pumps. The feed-water was pumped direct from the hot-well to the boiler. Taking $18.8 \mathrm{lbs}$, of steam per I.H.P. for the non-condensing, and $12.54 \mathrm{lbs}$. of steam for the triple-condensing engine, the economy in steam, due to condensing, is 33 per cent., the economy in cosl 30 per cent. The triple-condensing engiues had the advantage of being larger in size, and working at $17 \mathrm{lbs}$. higher boiler pressure, but as regards economy of fuel, the non-condensing engine had the advantage of the feed-heater. If 25 per cent. be taken as the saving in fuel due to condensing, when the best engine of each type, working at the same steam-pressures, is used, it will be very near the mark.

The Tables show that in Lighting Stations the non-condensing engines beat the condensing by 13 per cent. in fuel economy; this is the average over the whole country, $13+25=38$ per cent. to be accounted for to bring matters as they ought to be. Uneconomical, 
three-crank compound, tandem, condensing engines, largely used in Lighting Stations, no doubt account for some of this, and, as already shown, separate condensing plants are also steam wasters.

All non-condensing stations should bo fitted with efficient exhaust steam feed-heaters; their cost is comparatively small, and the saving in fuel is a clear 13 or 14 per cent. over cold feed. Feedheaters or economisers fixed in the boiler flues cool the waste gases, and to a certain extent spoil the draught. If this has to be made good by using steam-jets under the grates, the actual saving in fuel may be small. The best results are to be got by' employing both types of heater, the water entering the economiser at nearly boilingpoint. It is interesting to note that Edinburgh and Motherwell, the two most economical non-condensing stations given in Table 4 (pages 526-7), are both fitted with exhaust steam feed-heaters. About fifteen jears ago, the author fitted this type of heater on a condensing engine with good results. It is now the common practice in Americe to fit these heaters on condensing engines.

Engines in General.-The steam losses in electric generating engines are mostly due to valve or piston leakage; mainly the former. This applies more especially to condensing stations using engines fitted with piston valves; unless these are very carefully looked after, heavy leakage is likely to occur, the steam passing direct to the condenser. In non-condensing engines the exhaust pipe usually gives warning of leaky valves. Many station engineers do not appear to realise how serious this loss may be, and allow their piston-valves to run in a very leaky condition. Others give this matter most careful attention, and are well rewarded for their trouble; in fact, some of the most economical non-condensing stations are fitted throughout with piston-ralve engines, carefully looked after and kept steam-tight. Balanced slide-valves, and valves of the Corliss type, having some pressure on the back to keep them up to the port faces, require much less attention, and will run for many yoars practically stoam-tight.

Two recent tests of condensing engines (in different stations), fitted with piston-valves, disclosed the fact that they required over 45 lbs. and 50 lbs. of steam per kilowatt-hour respectively. When 
previously tested the consumption was about $30 \mathrm{lbs}$. per kilowatt in each case. Outwardly they appeared to be running as well as ever.

Coal to the value of $£ 839,613$ was used in the 177 stations given in the Tables, during the year under review. It is safe to essume that $£ 100,000$ was lost in engine leakages alone. There is no doubt it would pay to employ an engineer in each of the larger stations to do nothing else but make tests and report on fuel losses with a view to their remedy.

Boiler-Huse Plant.-There are great differences in the qualities of steam generated by boilers. Engine-builders find much less difficulty in getting their guarantee figures from the honest steam of the Lancashire or other cylindrical boiler than they do from the high-pressure "Scotch Mist" given off by the now fashionable box of water-pipes. In lighting stations there are great fluctuations in the demand for steam. Only those who really understand the "art" of firing, can appreciate the difficulty of working mechanical stokers with economy under such conditions. Extra grate area may be the salvation of the "tyro" at the higher loads, but with such men, it is fatal to economy at the lighter loads, especially when steam-jets to force the draught are used.

An efficient method of forced draught is a decided advantage in lighting stations, if it is properly used, for it enables steam to be maintained over the peak of the load. The latest system of Mr. Halpin's thermal storage is the ideal method of obtaining the same end. At light loads the steam-jet method of forcing dranght is often greatly mismanaged. A peep through the small side door, at the back of the grates, in a Babcock boiler, fitted with moving grates and forced draught, and carrying, say, half load, is often a revelation of how not to do it. One-third of the grate may be covered by a spouting volcano of fire, the remainder employed in carrying away dead ashes and clinker, allowing large quantities of cold air to pass, all of which has to be heated up to the temperature of the escaping gases. Under these conditions, a low flue temperature is no criterion of economy.

Some makers of steam-jet draught-producers only require 3 per cent. of the steam generated to supply their blowers; others state that they require 3 per cent., but their fittings are found to be large enough 
to pass four times that amount, even when the full rating of the boiler is taken. The average fireman, as a rule, goes for all he can get under any conditions; hence it comes that he is able to maintain steam under the conditions already described, and waste large quantities of fuel by heating up the great excess of air passing throngh the uncovered bars.

Some years ago the author made a steam-economy test of a large pumping plant in the Colonies. The boilers were fired with wood, and the dranght was got by means of steam-jets. It was found that they used 19 per cent. of the total steam generated. In a recent test of a modern stoker the numerous steam-jets used 12 per cent. of the total steam generated. By the use of fans, especially when heated air is used, good economy is obtainable. Feed-pumps and other boiler-house auxiliary engines require a considerable percentage of the steam generated. In condensing stations, better economy is obtained by utilizing the exhaust of the auxiliary engines in heating the food than working them as condensing engines. In lighting stations the losses in the ring steam main, with its numerous traps, is a serious consideration; it is seldom less than 5 per cent., and may be very much more if the traps are not kept in perfect order and properly regulated.

Superheating, especially when piston-valve engines are used, shows a good steam economy, but the saving in fuel is not so marked. In lighting stations its proper regulation is rather difficult. The extra wear and tear on the main engines, and on the superheaters themselves, are items that have to be considered.

The Station Engineer.- $\mathrm{He}$ should note that the average works costs for the non-condensing lighting stations, Table 1 (page 528)

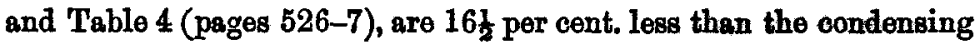
stations, given in Tables 3 and 6 . One explanation of this may be that less plant requires less looking after and less repairs; there is also less capital invested. But a well-managed condensing station with economical engines, fitted with their own condensers, \&c., is certainly the most economical type of station, especislly where trams are coupled with lighting. The figures already given prove that the great economy due to condensing may be frittered away by using unsuitable plant, and by careless management. In his specifications, 
he should insist that the total steam used by the main engine and its condensing plant be included in the guaranteed consumption; and that the evaporation of the boilers be taken in dry steam, delivered to the engine, and exclusive of steam used for various purposes in the working of the boiler. He would then be much better able to decide on the best type of plant to use.

Steam Turbines.-There are only four stations given in the Tables wholly using steam turbines:-Newcastle District, Table 5 (page 528), Scarborough, Cambridge, and Melton Mowbray, Table 6 (pages 529-530). Newcastle Station is the lowest, both in coal, and works costs; yet it is easily beaten by South Shields, a station with rastly less output, situated on the same river, and using tripleexpansion condensing engines, which comes out 35 per cent. less in fuel, and 25 per cent. lower in works costs than the turbine station.

Gas-Engine Stations.-In the costs Table of the "Electrical Times," particulars are given of only two gas-engine stations." Northwich, using "Mond" producer-gas, has an output of 104,000 units. Its fuel cost is 0.48 penny per unit; works costs are 1.48 per unit. This is handsomely beaten by Leigh, Table 4. (page 526), a station with a larger output, using non-condensing steam-engines, which comes out at 28 per cent. less in fuel, and 15 per cent. less in works costs.

Redditch, the other gas-engine station, using "Dowson" producer-gas, has an output of over 172,000 units; coal costs are 1.3 pence per unit, and works costs are $2 \cdot 61$ pence per unit. There are over a dozen smaller sized steam-engine stations given in the Tables that beat these results.

It is encouraging to note from recently published returns that the costs in various stations throughout the country show a decided tendency to improvement. This is partly owing to cheaper coal, but there is also a healthy rivalry between station engineers, stimulated no doubt, by the "Electrical Times" publishing their results. This should in time bring the electric generating stations into the front rank as regards economy of fuel.

If any remarks in this Paper assist the station engineer towards obtaining this better economy, the author will feel amply rewarded. 
TABLE 1.

Metropolitan Stations. Non-Condensing.

\section{Lighting ONLY.}

\begin{tabular}{|c|c|c|c|c|c|c|}
\hline & - & $\begin{array}{l}\text { Coal } \\
\text { per } \\
\text { Unit } \\
\text { sold. }\end{array}$ & $\begin{array}{l}\text { Units } \\
\text { sold. }\end{array}$ & $\begin{array}{l}\text { Units } x \\
\text { Coal Cost. }\end{array}$ & $\begin{array}{c}\text { Works } \\
\text { Costs } \\
\text { per Unit } \\
\text { sold. }\end{array}$ & $\begin{array}{l}\text { Units } x \\
\text { Works Costs } \\
\text { cost. }\end{array}$ \\
\hline Blackheath . & & $\underset{1 \cdot 22}{d .2}$ & 359,554 & $\begin{array}{c}d . \\
438,655\end{array}$ & $\begin{array}{c}d . \\
2 \cdot 18\end{array}$ & $\begin{array}{c}d . \\
783,827\end{array}$ \\
\hline Chelsea . & . & $0 \cdot 81$ & $2,011,150$ & $1,629,031$ & $1 \cdot 70$ & $3,418,955$ \\
\hline Islington . & . & $1 \cdot 16$ & $2,186,044$ & $2,535,811$ & 1.98 & $4,328,367$ \\
\hline Notting Hill! · & - & 0.84 & 947,491 & 795,892 & $1 \cdot 58$ & $1,497,035$ \\
\hline St. James's . & . & 0.86 & $5,842,496$ & $5,024,546$ & $1 \cdot 37$ & $8,004,219$ \\
\hline Shoreditch & . & $*_{1} \cdot 48$ & $2,247,055$ & $3,325,641$ & $2 \cdot 25$ & $5,055,873$ \\
\hline Sonthwark & . & $1 \cdot 35$ & 484,431 & 653,981 & $2 \cdot 29$ & $1,109,346$ \\
\hline Stepney . & . & 0.5 & $1,008,037$ & 504,018 & 0.81 & 816,509 \\
\hline Westminster. & . & $1 \cdot 04$ & $8,927,883$ & $9,284,998$ & $1 \cdot 59$ & $14,195,333$ \\
\hline Total & . & - & $24,014,141$ & $24,192,573$ & - & $39,209,464$ \\
\hline
\end{tabular}

$\frac{24,192,573}{24,014,141}=1.007$ pence per unit sold for Coal.

$\frac{39,209,464}{24,014,141}=1 \cdot 632$ pence per unit sold for Works Costs.

* Steam partly supplied by Refuse Destructor. 


\section{TABLE 2.}

Metropolitan Stations. Partly Condensing.

Lighting OnLy.

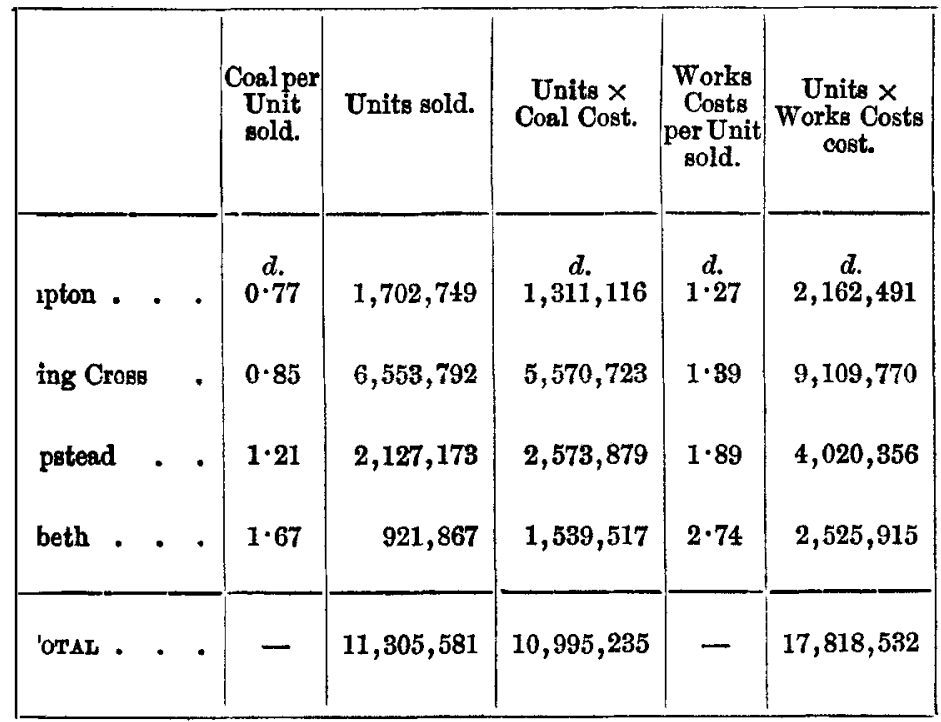

$\frac{10,995,235}{11,305,581}=0 \cdot 972$ pence per unit sold for Coal.

$\frac{17,818,582}{11,305,581}=1 \cdot 576$ pence per unit sold for Works Costs. 


\section{TABLE 3.}

\section{Metropolitan Stations. Condensing.}

\section{Lighting Only.}

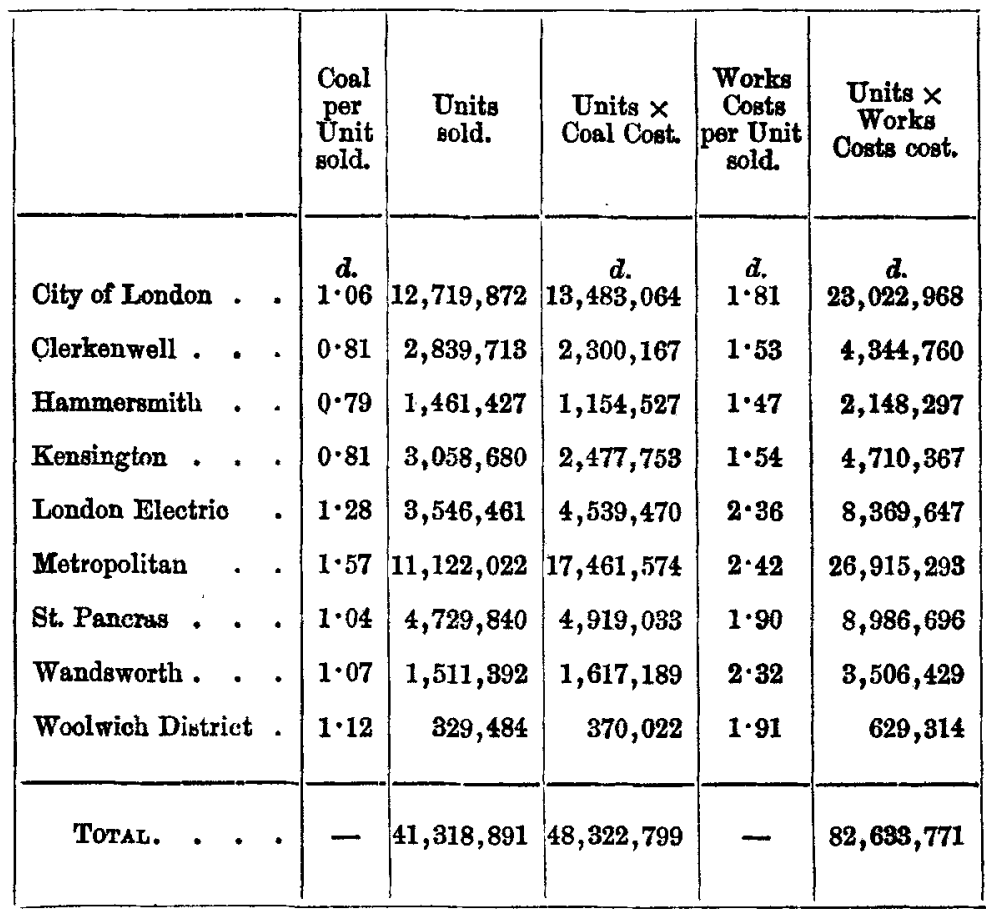

$\frac{48,322,799}{41,318,891}=1 \cdot 169$ pence per unit sold for Ooal.

$\frac{82,633,771}{41,318,891}=1.999$ pence per unit sold for Works Costs. 
LE 4 (continued on opposite page).

Provincial Non-Condensing Stations.

Lrghtrng ONLY.

\begin{tabular}{|c|c|c|c|c|c|c|}
\hline & & $\begin{array}{l}\text { Coal per } \\
\text { Unit } \\
\text { sold. }\end{array}$ & Units sold. & $\begin{array}{c}\text { Units } x \\
\text { Coal cost. }\end{array}$ & $\mid \begin{array}{c}\text { Works } \\
\text { Costs } \\
\text { perUnit } \\
\text { sold. }\end{array}$ & $\begin{array}{c}\text { Units } \times \\
\text { Works Costs } \\
\text { cost. }\end{array}$ \\
\hline $\begin{array}{l}\text { ystwyth } \\
\text { tley : } \\
\text { or. }\end{array}$ & : : & $\begin{array}{l}d . \\
1 \cdot 72 \\
1 \cdot 03 \\
2 \cdot 05\end{array}$ & $\begin{array}{l}78,815 \\
29,853 \\
86,820\end{array}$ & $\begin{array}{r}d . \\
135,561 \\
30,748 \\
177,981\end{array}$ & $\begin{array}{l}d . \\
2 \cdot 95 \\
2 \cdot 23 \\
3 \cdot 3\end{array}$ & $\begin{array}{c}d . \\
232,504 \\
66,583 \\
286,506\end{array}$ \\
\hline $\begin{array}{l}\text { iley } \\
\text { mhead } \\
\text { St. Edm }\end{array}$ & ands & $\begin{array}{l}0 \cdot 79 \\
0 \cdot 61 \\
1 \cdot 21\end{array}$ & $\begin{array}{r}200,112 \\
391,516 \\
73,042\end{array}$ & $\begin{array}{r}158,088 \\
238,824 \\
88,380\end{array}$ & $\begin{array}{l}1 \cdot 41 \\
1 \cdot 34 \\
2 \cdot 08\end{array}$ & $\begin{array}{l}882,157 \\
524,631 \\
151,927\end{array}$ \\
\hline $\begin{array}{l}\text { nsford } \\
\text { ngton. }\end{array}$ & : : & $\begin{array}{l}1 \cdot 79 \\
0.59 \\
0 \cdot 57\end{array}$ & $\begin{array}{r}82,906 \\
310,505 \\
162,563\end{array}$ & $\begin{array}{r}148,401 \\
183,197 \\
92,660\end{array}$ & $\begin{array}{l}2 \cdot 72 \\
1 \cdot 01 \\
1 \cdot 16\end{array}$ & $\begin{array}{l}225,504 \\
313,610 \\
188,573\end{array}$ \\
\hline $\begin{array}{l}\text { bury . } \\
\text { n . } \\
\text { surgh. }\end{array}$ & $: \dot{:}$ & $\begin{array}{l}0 \cdot 60 \\
1 \cdot 85 \\
0 \cdot 34\end{array}$ & $\begin{array}{r}263,076 \\
625,580 \\
7,760,307\end{array}$ & $\begin{array}{r}157,845 \\
1,157,323 \\
2,638,504\end{array}$ & $\begin{array}{l}1 \cdot 61 \\
3 \cdot 24 \\
0 \cdot 65\end{array}$ & $\begin{array}{r}423,552 \\
2,026,879 \\
5,044,199\end{array}$ \\
\hline $\begin{array}{l}r \text { stone } \\
2 .\end{array}$ & : : & $\begin{array}{l}1 \cdot 49 \\
1 \cdot 25 \\
0 \cdot 42\end{array}$ & $\begin{array}{l}311,160 \\
464,392 \\
409,397\end{array}$ & $\begin{array}{l}463,628 \\
580,490 \\
171,946\end{array}$ & $\begin{array}{l}2 \cdot 7 \\
1 \cdot 9 \\
1 \cdot 22\end{array}$ & $\begin{array}{l}840,132 \\
882,344 \\
499,464\end{array}$ \\
\hline $\begin{array}{c}\text { lock : } \\
\text { w. : } \\
\text {. }\end{array}$ & : & $\begin{array}{l}1 \cdot 00 \\
1 \cdot 58 \\
1 \cdot 15\end{array}$ & $\begin{array}{l}157,681 \\
183,908 \\
629,427\end{array}$ & $\begin{array}{l}157,681 \\
281,379 \\
723,741\end{array}$ & $\begin{array}{l}1 \cdot 75 \\
3 \cdot 02 \\
1 \cdot 8\end{array}$ & $\begin{array}{r}275,941 \\
555,402 \\
1,132,968\end{array}$ \\
\hline Wight & : : & $\begin{array}{l}0 \cdot 88 \\
1 \cdot 56 \\
0 \cdot 35\end{array}$ & $\begin{array}{l}403,144 \\
182,384 \\
151,048\end{array}$ & $\begin{array}{r}354,766 \\
284,519 \\
52,866\end{array}$ & $\begin{array}{l}1 \cdot 33 \\
2 \cdot 43 \\
1 \cdot 01\end{array}$ & $\begin{array}{l}536,181 \\
443,193 \\
152,558\end{array}$ \\
\hline $\begin{array}{l}\text { n: } \\
\text { rool Dist }\end{array}$ & rict. & $\begin{array}{l}0 \cdot 84 \\
0 \cdot 68 \\
1 \cdot 13\end{array}$ & $\begin{array}{l}511,765 \\
811,141 \\
126,689\end{array}$ & $\begin{array}{l}429,882 \\
551,575 \\
143,158\end{array}$ & $\begin{array}{l}1 \cdot 36 \\
1 \cdot 36 \\
2 \cdot 4\end{array}$ & $\begin{array}{r}696,000 \\
1,103,151 \\
304,053\end{array}$ \\
\hline $\begin{array}{l}\text { rindod } \\
Y . \quad\end{array}$ & : : & $\begin{array}{l}1 \cdot 35 \\
1 \cdot 03\end{array}$ & $\begin{array}{l}79,420 \\
99,205\end{array}$ & $\begin{array}{l}107,217 \\
102,181\end{array}$ & $\begin{array}{l}2 \cdot 8 \\
2 \cdot 28\end{array}$ & $\begin{array}{l}222,376 \\
226,187\end{array}$ \\
\hline Carried & over & - & $14,585,856$ & $9,612,541$ & - & $18,236,575$ \\
\hline
\end{tabular}


TABLE 4 (concluded from opposite page). Provincial Non-Condensing Stations.

Lighting OnLy.

\begin{tabular}{|c|c|c|c|c|c|}
\hline & $\begin{array}{l}\text { Cosl per } \\
\text { Unit } \\
\text { sold. }\end{array}$ & Units sold. & $\begin{array}{c}\text { Units } \times \text { Coal } \\
\text { cost. }\end{array}$ & $\begin{array}{c}\text { Works } \\
\text { Costs } \\
\text { perUnit } \\
\text { sold. }\end{array}$ & $\begin{array}{c}\text { Units } x \\
\text { Works Costs } \\
\text { cost. }\end{array}$ \\
\hline $\begin{array}{l}\text { Brought over } \\
\text { Motherwell : }\end{array}$ & $\begin{array}{c}d . \\
0 \cdot 46\end{array}$ & $\begin{array}{r}14,585,856 \\
166,860\end{array}$ & $\begin{array}{r}d . \\
9,612,541 \\
76,755\end{array}$ & $\begin{array}{c}d . \\
0 \cdot 98\end{array}$ & $\begin{array}{r}d . \\
18,236,575 \\
163,522\end{array}$ \\
\hline $\begin{array}{l}\text { Nolson : }: \\
\text { Newmarket : } \\
\text { Northampton : }\end{array}$ & $\begin{array}{l}0 \cdot 68 \\
1 \cdot 19 \\
0 \cdot 80\end{array}$ & $\begin{array}{r}106,189 \\
60,676 \\
286,890\end{array}$ & $\begin{array}{r}72,208 \\
72,204 \\
229,512\end{array}$ & $\begin{array}{l}2 \cdot 15 \\
2 \cdot 1 \\
1 \cdot 7\end{array}$ & $\begin{array}{l}228,306 \\
127,419 \\
487,713\end{array}$ \\
\hline 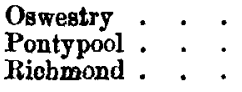 & $\begin{array}{l}1 \cdot 37 \\
0 \cdot 75 \\
1 \cdot 19\end{array}$ & $\begin{array}{r}50,649 \\
70,491 \\
217,789\end{array}$ & $\begin{array}{r}69,389 \\
52,868 \\
259,168\end{array}$ & $\begin{array}{l}2 \cdot 87 \\
1 \cdot 88 \\
1 \cdot 96\end{array}$ & $\begin{array}{l}145,362 \\
132,523 \\
426,866\end{array}$ \\
\hline $\begin{array}{l}\text { Shrewsbury } \\
\text { St. } \\
\text { Stirlinges'. : : : }\end{array}$ & $\begin{array}{l}0.99 \\
0.57 \\
0.99\end{array}$ & $\begin{array}{r}249,263 \\
98,369 \\
111,369\end{array}$ & $\begin{array}{r}246,770 \\
56,070 \\
110,255\end{array}$ & $\begin{array}{l}1 \cdot 65 \\
1 \cdot 39 \\
1 \cdot 98\end{array}$ & $\begin{array}{l}411,283 \\
136,732 \\
220,510\end{array}$ \\
\hline $\begin{array}{l}\text { Stookton : : } \\
\text { Watford : } \\
\text { West Hartlepool : }\end{array}$ & $\begin{array}{l}0.84 \\
1.94 \\
0.61\end{array}$ & $\begin{array}{l}205,322 \\
290,947 \\
268,523\end{array}$ & $\begin{array}{l}172,470 \\
561,437 \\
163,799\end{array}$ & $\begin{array}{l}2 \cdot 2 \\
3 \cdot 05 \\
1 \cdot 69\end{array}$ & $\begin{array}{l}451,708 \\
887,388 \\
453,803\end{array}$ \\
\hline $\begin{array}{l}\text { Wimbledon } \\
\text { Winchester : } \\
\text { Windsor : }\end{array}$ & $\begin{array}{l}* 1 \cdot 18 \\
1 \cdot 36 \\
1 \cdot 24\end{array}$ & $\begin{array}{l}806,280 \\
192,385 \\
366,534\end{array}$ & $\begin{array}{l}951,410 \\
261,643 \\
454,502\end{array}$ & $\begin{array}{l}1 \cdot 7 \\
1 \cdot 69 \\
2 \cdot 01\end{array}$ & $\begin{array}{r}1, \mathbf{3 7 0}, 676 \\
325,130 \\
796,733\end{array}$ \\
\hline Wycombe & $1 \cdot 17$ & 295,727 & 346,000 & $1 \cdot 66$ & 195,179 \\
\hline TOTAL . & - & $18,430,119$ & $13,772,001$ & - & $25,137,428$ \\
\hline
\end{tabular}

$\frac{13,772,001}{18,430,119}=0 \cdot 747$ pence per units sold for Coal.

$\frac{25,137,428}{18,430,119}=1 \cdot 363$ pence per nnit sold for Works Costs.

* Steam partly supplied by Refuse I)estructor. 


\section{TABLE 5.}

\section{Provincial Partly-Condensing Stations.}

\section{LightiNG ONLY.}

\begin{tabular}{|c|c|c|c|c|c|}
\hline & $\begin{array}{l}\text { Coal per } \\
\text { Unit } \\
\text { sold. }\end{array}$ & Units sold. & $\begin{array}{l}\text { Units } \times \\
\text { Coal Cost. }\end{array}$ & $\begin{array}{c}\text { Works } \\
\text { Costs } \\
\text { per Unit } \\
\text { sold. }\end{array}$ & $\begin{array}{c}\text { Units } \times \\
\text { Works Costs } \\
\text { cost. }\end{array}$ \\
\hline Altrincham - . & $\stackrel{d .}{1 \cdot 83}$ & 354,615 & $\begin{array}{l}d . \\
648,945\end{array}$ & $\begin{array}{c}d . \\
\mathbf{3} \cdot 76\end{array}$ & $\begin{array}{c}d . \\
1,333,352\end{array}$ \\
\hline Belfast - . & $0 \cdot 61$ & $1,206,699$ & 736,086 & $1 \cdot 27$ & $1,532,507$ \\
\hline Birmingham . . & $1 \cdot 00$ & $3,391,099$ & $3,391,099$ & $1 \cdot 82$ & $6,171,800$ \\
\hline Bournemouth & $1 \cdot 04$ & $1,003,401$ & $1,043,537$ & $1 \cdot 77$ & $1,776,019$ \\
\hline Cardiff . . . & 0.67 & $1,005,763$ & 673,861 & $1 \cdot 46$ & $1,468,413$ \\
\hline Coventry . . . & $0 \cdot 80$ & 404,968 & 323,974 & $1 \cdot 8$ & 728,942 \\
\hline Eastbourne . . & $1 \cdot 82$ & 447,295 & 814,076 & $2 \cdot 74$ & $1,225,588$ \\
\hline Glasgow . . & $0 \cdot 36$ & $9,282,043$ & $3,341,535$ & $0 \cdot 95$ & $8,817,940$ \\
\hline Guildford & $1 \cdot 97$ & 99,150 & 195,325 & $2 \cdot 78$ & 275,637 \\
\hline Middlesbrough . & $0 \cdot 60$ & 281,332 & 170,599 & $1 \cdot 36$ & 386,691 \\
\hline Morecambe & $1 \cdot 28$ & 202,967 & 259,809 & $2 \cdot 61$ & 529,743 \\
\hline $\begin{array}{r}\text { Newcastle } \\
\text { District , . }\end{array}$ & $0 \cdot 69$ & $1,619,671$ & $1,117,572$ & $1 \cdot 22$ & $1,975,998$ \\
\hline Preston . & $0 \cdot 51$ & 912,142 & 465,192 & 0.92 & 839,170 \\
\hline Tunbridge Wells & $1 \cdot 5$ & 659,000 & 988,500 & $1 \cdot 98$ & $1,304,820$ \\
\hline TOTAL. & - & $20,873,145$ & $14,170,110$ & - & $28,366,620$ \\
\hline
\end{tabular}

$\frac{14,170,110}{20,873,145}=0.678$ pence per unit sold for Coal.

$\frac{28,366,620}{20,873,145}=1 \cdot 359$ pence per unit sold for Works Costs. 
JUIX 1903. FUEL ECONOMY IN GHNERATING STATIONS.

TABLE 6 (continued on next page).

Provincial Condensing Stations.

Lighting Onlt.

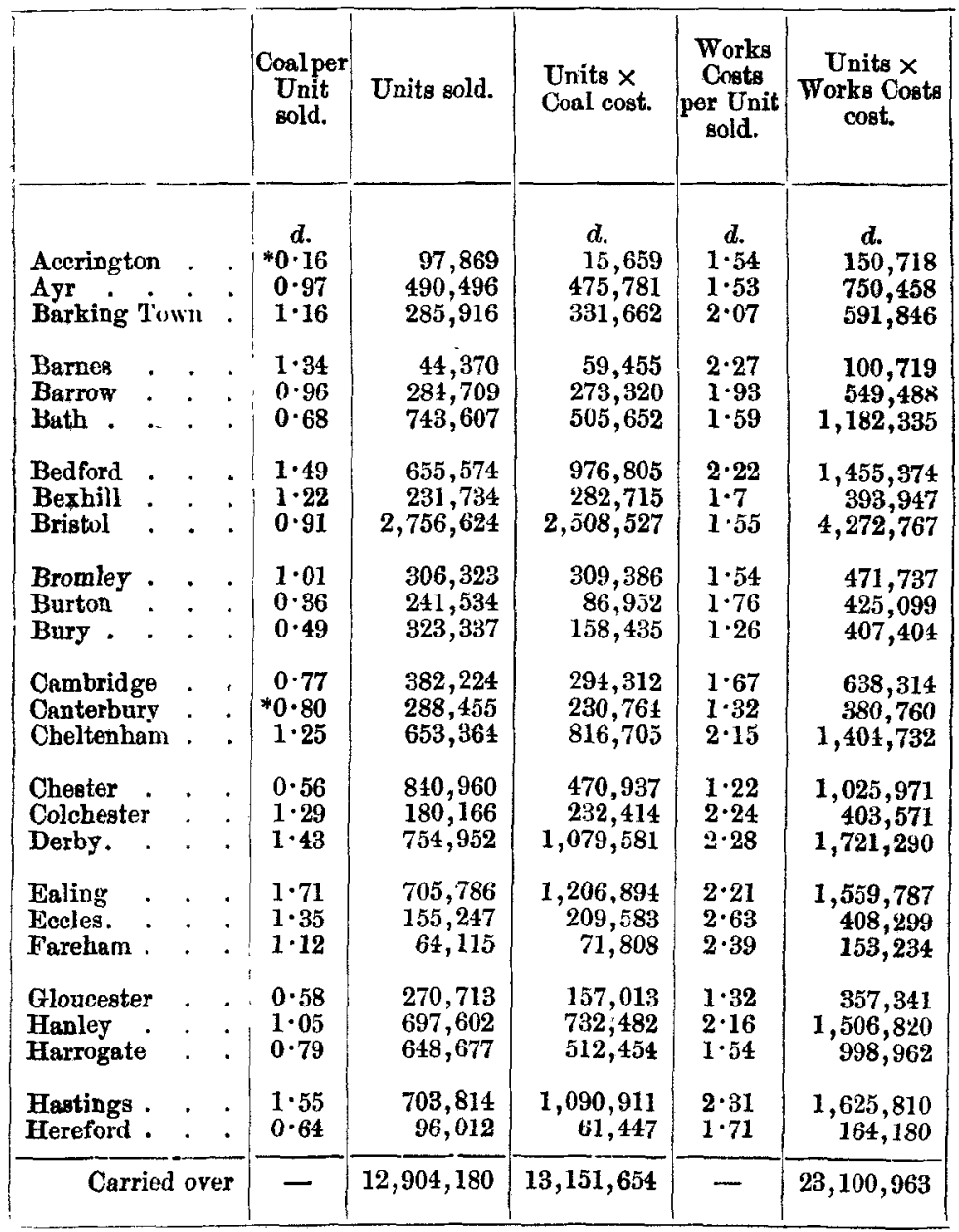

* Steam partly supplied by Refuse Destructor. 
TABLE 6 (continued on opposite page).

Provincial Condensing Stations.

Lrahting OnLY.

\begin{tabular}{|c|c|c|c|c|c|}
\hline & $\begin{array}{l}\text { Coal per } \\
\text { Unit } \\
\text { sold. }\end{array}$ & Units sold. & $\begin{array}{l}\text { Units } \times \\
\text { Cosl Cost. }\end{array}$ & $\begin{array}{c}\text { Works } \\
\text { Costs } \\
\text { per Unit } \\
\text { sold. }\end{array}$ & $\begin{array}{l}\text { Units } \times \\
\text { Works Costs } \\
\text { cost. }\end{array}$ \\
\hline $\begin{array}{l}\text { Brought over } \\
\text { Huddersfield }\end{array}$ & $\begin{array}{c}d . \\
0 \cdot 51\end{array}$ & $\begin{array}{r}12,904,180 \\
1,179,849\end{array}$ & $\begin{array}{r}d . \\
13,151,654 \\
601,722\end{array}$ & $\begin{array}{c}d . \\
1 \cdot 14\end{array}$ & $\begin{array}{c}d \\
23,100,963 \\
1,345,027\end{array}$ \\
\hline $\begin{array}{l}\text { Hull } \\
\text { King's Lyin: : } \\
\text { Kingston . : }\end{array}$ & $\begin{array}{l}0.84 \\
0.59 \\
2.46\end{array}$ & $\begin{array}{r}1,490,099 \\
286,679 \\
346,332\end{array}$ & $\begin{array}{r}1,251,683 \\
169,140 \\
851,976\end{array}$ & $\begin{array}{l}1 \cdot 74 \\
1 \cdot 26 \\
3 \cdot 35\end{array}$ & $\begin{array}{r}2,592,772 \\
361,215 \\
1,160,212\end{array}$ \\
\hline $\begin{array}{l}\text { Lancaster. . : } \\
\text { Loods . : : } \\
\text { Leicester. . }\end{array}$ & $\begin{array}{r}* 0.82 \\
0 \cdot 27 \\
0.55\end{array}$ & $\begin{array}{r}341,536 \\
3,055,165 \\
1,042,302\end{array}$ & $\begin{array}{l}280,059 \\
824,894 \\
573,266\end{array}$ & $\begin{array}{l}1 \cdot 79 \\
0 \cdot 62 \\
1 \cdot 34\end{array}$ & $\begin{array}{r}611,349 \\
1,894,202 \\
1,396,684\end{array}$ \\
\hline $\begin{array}{l}\text { Lincoln . } \\
\text { Llandudno } \\
\text { Melton Mowbray }\end{array}$ & $\begin{array}{r}0.58 \\
* 0.69 \\
0.93\end{array}$ & $\begin{array}{r}263,216 \\
279,292 \\
43,742\end{array}$ & $\begin{array}{r}152,665 \\
192,711 \\
40,680\end{array}$ & $\begin{array}{l}1 \cdot 35 \\
1 \cdot 35 \\
2 \cdot 19\end{array}$ & $\begin{array}{r}355,341 \\
377,044 \\
95,794\end{array}$ \\
\hline $\begin{array}{l}\text { Newport : }: \text { : } \\
\text { Norwich : }: \text { : } \\
\text { Oxford }: \text { : }\end{array}$ & $\begin{array}{l}0.50 \\
0.85 \\
0.89\end{array}$ & $\begin{array}{r}578,275 \\
1,332,109 \\
569,561\end{array}$ & $\begin{array}{r}289,137 \\
1,132,292 \\
506,909\end{array}$ & $\begin{array}{l}1 \cdot 39 \\
1 \cdot 59 \\
2 \cdot 21\end{array}$ & $\begin{array}{r}803,802 \\
2,118,053 \\
1,258,729\end{array}$ \\
\hline $\begin{array}{l}\text { Paisley : : } \\
\text { Portsmouth : } \\
\text { Prescot : }\end{array}$ & $\begin{array}{l}1 \cdot 41 \\
1 \cdot 11 \\
0 \cdot 99\end{array}$ & $\begin{array}{r}370,521 \\
1,847,790 \\
215,231\end{array}$ & $\begin{array}{r}522,421 \\
2,051,046 \\
213,078\end{array}$ & $\begin{array}{l}2 \cdot 92 \\
1 \cdot 7 \\
1 \cdot 7\end{array}$ & $\begin{array}{r}1,081,921 \\
3,141,243 \\
365,892\end{array}$ \\
\hline $\begin{array}{l}\text { Rathmines : } \\
\text { Reading : } \\
\text { Rochdale : }\end{array}$ & $\begin{array}{l}0.85 \\
0.89 \\
0.89\end{array}$ & $\begin{array}{r}362,552 \\
464,580 \\
91,632\end{array}$ & $\begin{array}{r}308,169 \\
413,476 \\
81,552\end{array}$ & $\begin{array}{l}1 \cdot 34 \\
1 \cdot 42 \\
2 \cdot 24\end{array}$ & $\begin{array}{l}485,819 \\
659,703 \\
205,255\end{array}$ \\
\hline $\begin{array}{l}\text { Salford . . } \\
\text { Salisbury : } \\
\text { Scarborough : }\end{array}$ & $\begin{array}{l}1 \cdot 35 \\
0 \cdot 46 \\
1 \cdot 31\end{array}$ & $\begin{array}{l}716,355 \\
150,222 \\
379,743\end{array}$ & $\begin{array}{r}967,079 \\
69,106 \\
497,463\end{array}$ & $\begin{array}{l}2 \cdot 87 \\
1 \cdot 21 \\
2 \cdot 1\end{array}$ & $\begin{array}{r}2,055,938 \\
181,768 \\
797,460\end{array}$ \\
\hline $\begin{array}{l}\text { Sheffield } \\
\text { South Shields : } \\
\text { Stafford . }\end{array}$ & $\begin{array}{l}0.46 \\
0.44 \\
0.62\end{array}$ & $\begin{array}{r}2,487,584 \\
985,646 \\
245,803\end{array}$ & $\begin{array}{r}1,144,428 \\
433,684 \\
152,397\end{array}$ & $\begin{array}{l}1 \cdot 0 \\
0 \cdot 92 \\
1 \cdot 77\end{array}$ & $\begin{array}{r}2,487,584 \\
906,794 \\
435,171\end{array}$ \\
\hline Cerried over & - & $32,029,996$ & $26,872,687$ & 一 & $50,275,735$ \\
\hline
\end{tabular}

- Steam partly supplied by Refuse Dest ructor. 
TABLE 6 (concluded from page 529).

\section{Provincial Condensing Stations.}

\section{Lighting Only.}

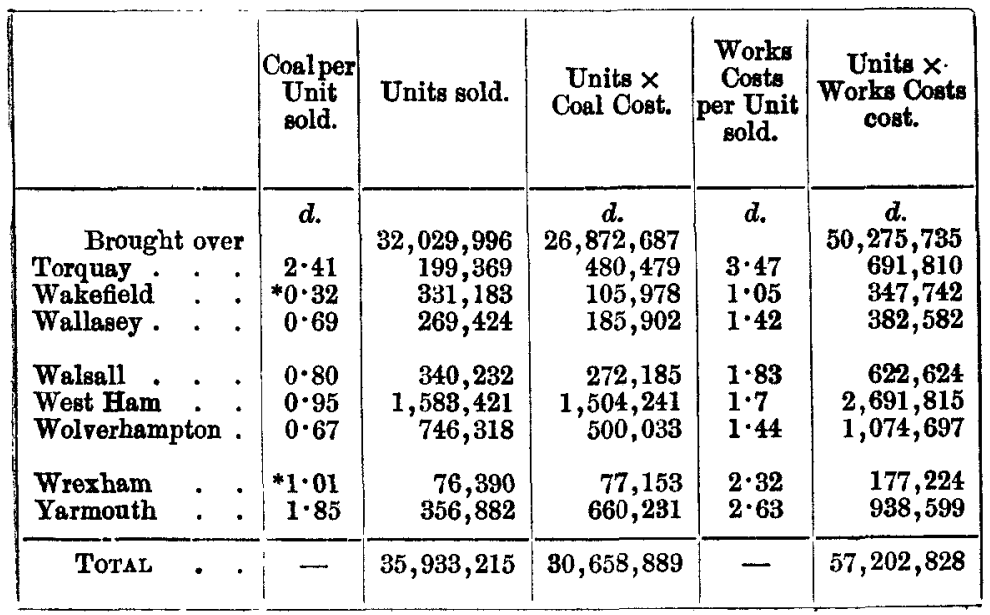

$\frac{30,658,889}{35,933,215}=0.853$ pence per unit sold for Coal.

$\frac{57,202,828}{35,933,215}=1.591$ penoe per unit sold for Works Costs.

* Steam partly surplied by Refuse Destructor. 


\section{TABLE 7.}

\section{Lighting and Tramways.}

Non-Condensing.

\begin{tabular}{|c|c|c|c|c|c|c|}
\hline & & $\begin{array}{c}\text { Coal } \\
\text { per } \\
\text { Unit } \\
\text { sold or } \\
\text { gener- } \\
\text { ated. }\end{array}$ & $\begin{array}{l}\text { Units } \\
\text { generated } \\
\text { or sold. }\end{array}$ & $\begin{array}{c}\text { Units } \times \text { Coal } \\
\text { cost. }\end{array}$ & $\begin{array}{c}\text { Works } \\
\text { Costs } \\
\text { per Unit } \\
\text { sold or } \\
\text { generated. }\end{array}$ & $\begin{array}{l}\text { Units } \times \\
\text { Works Costs } \\
\text { cost. }\end{array}$ \\
\hline Blackpool & - . & $\begin{array}{c}d . \\
1 \cdot 01\end{array}$ & $2,018,132$ & $\underset{2,038,313}{d .}$ & $\begin{array}{c}d \cdot \\
1 \cdot 82\end{array}$ & $\underset{3,673,000}{d .}$ \\
\hline Nottingham & . . & 0.46 & $4,094,897$ & $1,883,652$ & 0.81 & $3,316,866$ \\
\hline Southamptor & . & $0 \cdot 94$ & $1,430,222$ & $1,344,408$ & $1 \cdot 67$ & $2,388,470$ \\
\hline Sinckport & - . & 0.62 & 425,175 & 263,608 & $1 \cdot 43$ & 608,000 \\
\hline Total & - $\quad$. & - & $7,968,426$ & $5,529,981$ & - & $9,986, \mathbf{3 3 6}$ \\
\hline
\end{tabular}

$\frac{5,529,981}{7,968,426}=0.693$ pence per unit sold or generated for Coal.

$\frac{9,986,336}{7,968,426}=1 \cdot 253$ pence per unit sold or generated for Works Costs. 


\section{TABLE 8.}

\section{Lighting and Tramways.}

Pantly Condenging.

\begin{tabular}{|c|c|c|c|c|c|c|}
\hline & & $\begin{array}{l}\text { Coal } \\
\text { per } \\
\text { Unit } \\
\text { sold or } \\
\text { gener- } \\
\text { ated. }\end{array}$ & $\begin{array}{l}\text { Units } \\
\text { generated } \\
\text { or sold. }\end{array}$ & $\begin{array}{l}\text { Units } x \\
\text { Coal cost. }\end{array}$ & $\begin{array}{l}\text { Works } \\
\text { Costs } \\
\text { per Unit } \\
\text { generated } \\
\text { or sold. }\end{array}$ & $\begin{array}{l}\text { Units } \times \\
\text { Works Costs } \\
\text { cost. }\end{array}$ \\
\hline Aberdeen & - . & $\begin{array}{c}d . \\
0 \cdot 66\end{array}$ & $1,546,569$ & $\begin{array}{c}d . \\
1,020,735\end{array}$ & $\begin{array}{r}d . \\
1 \cdot 1\end{array}$ & $\stackrel{d .}{1,701,225}$ \\
\hline Ashton . & $\cdot \cdot$ & $0 \cdot 69$ & 977,044 & 674,160 & $1 \cdot 18$ & $1,152,911$ \\
\hline Blackburn & . $\cdot$ & 0.71 & $2,002,141$ & $1,421,520$ & $1 \cdot 09$ & $2,182,333$ \\
\hline Bolton . & $\cdot$. & $0 \cdot 44$ & $3,120,709$ & $1,373,111$ & $0 \cdot 80$ & $2,496,567$ \\
\hline Liverpool & . . & $0 \cdot 50$ & $20,018,142$ & $10,009,071$ & $0 \cdot 81$ & $16,214,695$ \\
\hline Bunderland & . & $0 \cdot 73$ & $2,375,557$ & $1,734,156$ & $1 \cdot 11$ & $2,636,868$ \\
\hline ToraL & - $\cdot$ & - & $30,040,162$ & $16,232,753$ & - & $26,384,599$ \\
\hline
\end{tabular}

$\frac{16,232,753}{30,040,162}=0.540$ pence per unit sold or generated for Coal.

$\frac{26,384,599}{30,040,162}=0.878$ pence per unit sold or generated for Works Cost. 


\section{TABLE 9.}

\section{Lighting and Tramways.}

Condinging.

\begin{tabular}{|c|c|c|c|c|c|c|}
\hline & & $\begin{array}{c}\text { Coal } \\
\text { per } \\
\text { Unit } \\
\text { sold or } \\
\text { gener- } \\
\text { ated. }\end{array}$ & $\begin{array}{l}\text { Units } \\
\text { generated } \\
\text { or sold. }\end{array}$ & $\begin{array}{c}\text { Units } \times \\
\text { Coul cost. }\end{array}$ & $\begin{array}{c}\text { Works } \\
\text { Costs } \\
\text { per Unit } \\
\text { sold or } \\
\text { generated. }\end{array}$ & $\begin{array}{c}\text { Units } \times \\
\text { Works Costs } \\
\text { cost. }\end{array}$ \\
\hline $\begin{array}{l}\text { Bootle } \\
\text { Bradford. } \\
\text { Brighton }\end{array}$ &. & $\begin{array}{c}d . \\
0 \cdot 59 \\
0 \cdot 39 \\
0 \cdot 74\end{array}$ & $\begin{array}{l}1,327,432 \\
4,901,172 \\
4,860,430\end{array}$ & $\begin{array}{r}d . \\
783,181 \\
1,911,457 \\
3,596,718\end{array}$ & $\begin{array}{c}d . \\
0 \cdot 90 \\
0 \cdot 76 \\
1 \cdot 43\end{array}$ & $\begin{array}{c}d . \\
1,194,688 \\
3,724,890 \\
6,950,414\end{array}$ \\
\hline $\begin{array}{l}\text { Burnley : } \\
\text { Carlisle : } \\
\text { Cork . }\end{array}$ & $: \quad:$ & $\begin{array}{l}0 \cdot 40 \\
0 \cdot 71 \\
0 \cdot 32\end{array}$ & $\begin{array}{r}505,736 \\
494,475 \\
1,701,996\end{array}$ & $\begin{array}{l}202,294 \\
351,077 \\
546,638\end{array}$ & $\begin{array}{l}1 \cdot 07 \\
1 \cdot 61 \\
0 \cdot 61\end{array}$ & $\begin{array}{r}541,137 \\
796,104 \\
1,038,217\end{array}$ \\
\hline $\begin{array}{l}\text { Croydon . } \\
\text { Dover : } \\
\text { Dundee : }\end{array}$ & 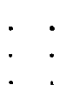 & $\begin{array}{l}0.92 \\
1.21 \\
0.82\end{array}$ & $\begin{array}{r}1,810,387 \\
781,809 \\
1,041,800\end{array}$ & $\begin{array}{r}1,665,556 \\
969,443 \\
851,276\end{array}$ & $\begin{array}{l}1 \cdot 34 \\
2 \cdot 13 \\
1 \cdot 33\end{array}$ & $\begin{array}{l}2,425,918 \\
1,665,253 \\
1,385,594\end{array}$ \\
\hline $\begin{array}{l}\text { Grimsby. } \\
\text { Halifax : } \\
\text { Manchester }\end{array}$ & : $:$ & $\begin{array}{l}0.81 \\
0.48 \\
0.41\end{array}$ & $\begin{array}{r}292,094 \\
2,557,548 \\
10,502,299\end{array}$ & $\begin{array}{r}245,358 \\
1,227,623 \\
4,305,912\end{array}$ & $\begin{array}{l}1 \cdot 46 \\
1 \cdot 09 \\
1 \cdot 15\end{array}$ & $\begin{array}{r}426,457 \\
2,787,727 \\
12,077,6 \pm 3\end{array}$ \\
\hline $\begin{array}{l}\text { Newcastle } \\
\text { Oldbam } \\
\text { Plymouth }\end{array}$ & $\dot{:}:$ & $\begin{array}{l}0.48 \\
0.71 \\
1.17\end{array}$ & $\begin{array}{r}2,562,383 \\
1,042,055 \\
832,433\end{array}$ & $\begin{array}{r}1,229,943 \\
739,859 \\
973,948\end{array}$ & $\begin{array}{l}0 \cdot 94 \\
1 \cdot 33 \\
1 \cdot 72\end{array}$ & $\begin{array}{l}2,408,640 \\
1,385,933 \\
1,431,788\end{array}$ \\
\hline $\begin{array}{l}\text { Southport } \\
\text { St. Helens } \\
\text { Taunton. }\end{array}$ & $: \quad:$ & $\begin{array}{l}0 \cdot 49 \\
0 \cdot 35 \\
1 \cdot 11\end{array}$ & $\begin{array}{r}1,462,407 \\
910,736 \\
885,907\end{array}$ & $\begin{array}{l}716,579 \\
318,757 \\
428,356\end{array}$ & $\begin{array}{l}0 \cdot 97 \\
0 \cdot 82 \\
1 \cdot 63\end{array}$ & $\begin{array}{r}1,418,534 \\
746,803 \\
629,028\end{array}$ \\
\hline $\begin{array}{l}\text { Tynemoutl } \\
\text { Whitehavin } \\
\text { Wigan . }\end{array}$ & $\therefore:$ & $\begin{array}{l}0.74 \\
0.7 \\
0.45\end{array}$ & $\begin{array}{l}449,467 \\
228,887 \\
579,904\end{array}$ & $\begin{array}{l}332,605 \\
160,220 \\
260,956\end{array}$ & $\begin{array}{l}1 \cdot 41 \\
1 \cdot 44 \\
1 \cdot 13\end{array}$ & $\begin{array}{l}633,748 \\
329,597 \\
655,291\end{array}$ \\
\hline Worcester & . & $0 \cdot 85$ & 662,569 & 563,183 & $1 \cdot 45$ & 960,725 \\
\hline Total & - & - & $39,893,928$ & $22,383,972$ & 一 & $45,614,129$ \\
\hline
\end{tabular}

$\frac{22,383,972}{39,893,928}=0 \cdot 561$ pence per unit suld or generated for Coal.

$\frac{45,614,129}{34,893,928}=1 \cdot 143$ pence per unit sold or generated for Works Costs. 
TABLE 10.

Electric Tramways.

Condengina.

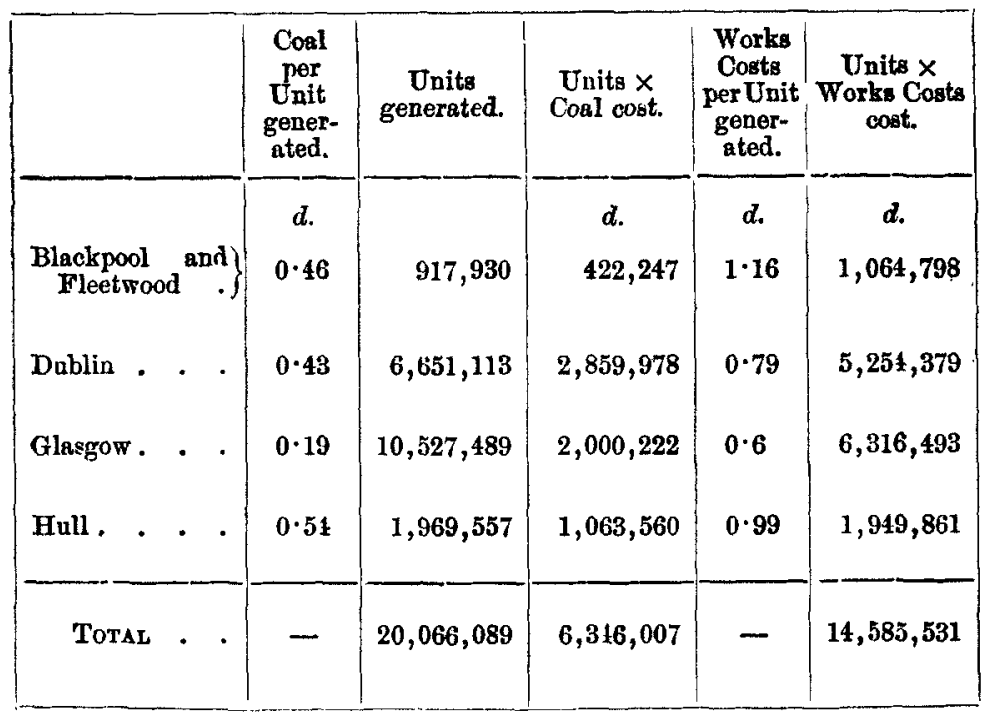

$\frac{6,346,007}{20,066,089}=0 \cdot 316$ per unit generated for Coal.

$\frac{14,585,531}{20,066,089}=0 \cdot 721$ per unit generated for Worki Costs. 


\section{TABLE 11.}

\section{Elsetric Railways.}

\section{Condensing.}

\begin{tabular}{|c|c|c|c|c|c|}
\hline & $\begin{array}{c}\text { Coal } \\
\text { per Unit } \\
\text { gener- } \\
\text { ated. }\end{array}$ & $\begin{array}{c}\text { Units } \\
\text { generated. }\end{array}$ & $\begin{array}{c}\text { Units } x \\
\text { Coal cost. }\end{array}$ & $\begin{array}{c}\text { Works } \\
\text { Costs per } \\
\text { Unit } \\
\text { gener- } \\
\text { ated. }\end{array}$ & $\begin{array}{l}\text { Unifs } \times \\
\text { Works Costs } \\
\text { cost. }\end{array}$ \\
\hline Central London & $\begin{array}{c}d . \\
0.38\end{array}$ & $16,753,292$ & $\begin{array}{c}d . \\
6,366,250\end{array}$ & $\begin{array}{c}d . \\
0.92\end{array}$ & $\begin{array}{c}d . \\
15,413,028\end{array}$ \\
\hline $\left.\begin{array}{c}\text { City and South } \\
\text { London } \\
.\end{array}\right\}$ & 0.28 & $4,850,420$ & $1,358,117$ & 0.94 & $4,559,394$ \\
\hline $\begin{array}{c}\text { Liverpool Over-) } \\
\text { head } .\end{array}$ & $0 \cdot 24$ & $4,910,28 \tilde{5}$ & $1,178,468$ & 0.83 & $4,075,536$ \\
\hline Total . & - & $26,513,997$ & $8,902,835$ & - & $24,047,958$ \\
\hline
\end{tabular}

$\frac{8,902,835}{26,513,997}=0 \cdot 335:$ per unit generated for Coal.

$\frac{24,047,958}{26,513,997}=0 \cdot 907$ per unit generated for Works Costs.

The Paper is illustrated by 1 Figure in the letterpress. 


\section{Discussion.}

Mr. MoLaren said the Puper had been written deliberately to promote discussion, so he hoped the members would not be spering in their oriticisms. Before the discussion commenced he wished to emphasise the last three lines in paragraph-3 (page 511), namely, "The price paid for coal varies largely, and different systems of supply are used. All these considerations affect the costs." Ho had taken the whole of the stations in the conntry, so that the average cost of fuel might be got throughont the country. Metropolitan statione were kept separate, and compared with metropolitan, as he presumed they paid the same for coal and labour in all these stations. The different systems of supply was a very important point, and he hoped that some of the electrical members present would throw some light on their effect, so far as works costs were concerned. The losses in the mains were debited to the generating plant in all lighting stations that measured the ourrent at the consumers' meters. Trouble would heve been saved if the current had been metered at the stations, and comparisons of the merits of the varions generating plants could have been made much easier. Metering the current at the consumers' premises, however, emphasised the fuct that there must be a great reduction in the costs of generating and distributing current, if central power stations were to compete for mill and factory driving.

Mr. Ade Clark in his Paper had compared the most eoonomical oil-ergine with a steam-engine that no self-respecting power-user would keop for long on his premises, and he considered that adrocates of central power-stations were doing very much the same thing. They compared the best they could put down with old antiquated engines, thus making a good comparison; but he would like some of the mill-engine builders to give those engineers a few figures of what they would have to compete with, if they wished to get the driving of mills or factories of any size. The wages of the engine men was a large factor in a small porver plant; it was not so important when one got to 100 H.P. and upwards; in one or two cases that he had investigated, the mill engines were generating 
(Mr. MrcLaren.)

their power on the premises at three-eighths of a penny per I.H.P., including interest and depreciation, etc. He considered that the best oil-engine should be compared with the best steam-engine; a gentleman should be compared with a gentleman, not with a rogue. With regard to gas-engines in central stations, he hoped that some of the manufacturers of gas-engines would shed some light on how it was that, with their highly perfected engines, they were not getting any better results, if as good, as the steam-engine. Costs per unit generated in central stations fitted with turbines did not show up better than the steam-engine stations. The figures he had given were taken from actual work. The steam-engine makers, gas-engine makers, and turbine makers were all pouring their most economical engines and prime movers into the capacious maw of the central station, and how was it that they did not get any better results out of the central station? He thought, perhaps, if they were to try two or three highly economical Diesel engines they might give out a better economy, but as jet they had not been tried. So far as gas-engines, steam-turbines, and steam-engines were concerned, the cost per unit at the consumers' meters were high out of all reason.

The President was sure it would be the pleasure of the members to convey their thanks to Mr. MeLaren for his most interesting Paper; he had thrown down the gauntlet most handsomely.

Mr. DrdtTt Halpin thanked the anthor for his kindly reference to himself in connection with boilers and Thermal Storage. Before proceeding to deal with the subject of the Paper, he wished to give one or two figures of comparison between gas and steam-engines which might be of use to the members, which came under his notice within the last fortnight. The gas plant was quite new, having been put down last year with all the latest improvements. The engines were of 250 H.P., and very accurate accounts were kept of what they were indicating, braking, and burning. Close by them in the same works there was a steamengine, and the owners noted carefully for a month what that steam-engine was doing. The engine was a tandem compound with 
a jet condenser, was of about 80 H.P., working at 60 lbs. pressure, which it had been doing during the last 28 years. The boilers that were driving it had been working for 32 years at $60 \mathrm{lbs}$. pressure. The gas-engine used $0.89 \mathrm{lb}$. of coal; the steam-engine used 3 lbs. of coal. But that was not the end of the story. The coal for the gas-engine cost 238. a ton; the, coal for the steam-engine cost 6s. $6 d$. a ton, so that, if it were taken out in money, the gasengine was using 5 per cent. more money than the engine 28 years old. This charge was for fuel only, and was exclusive of repairs, which were very heary in the case of the gas-engine plant.

Coming to the subject of the Paper, it might possibly be interesting to give some particulars of what was being done at the present moment. For a good many of the figures he intended to give he had to thank Mr. Miller, the engineer of the joint atation of the Kensington and Notting Hill Co. at Shepherd's Bush. That Company was making polyphase current at 5,000 volts, and sending it down to town where it was transformed. The full line, Plate 14, showed the load curve for the maximum load in January 1903, reaching about $2,000 \mathrm{kw}$, and the maximum load for January 1904, reaching $2,750 \mathrm{kw}$. The stations were increasing their load very fast, and the engineers found they would have to provide for an additional 1,200 I.H.P. in the coming winter. That 1,200 H.P. Was shown by the difference in the height between the top and bottom loed curve lines, and was built up from the result of their previous experience in their extensions. The dotted lines showed either cosl or thermal units put jinto the boilers. When they wore working in the old ordinary wryathe coar curve would be the top one for 1904, and with Thermal Storage in use if would be the lower one, the hatched area showing the amount of heat stored in the Thermal Storage vessels. The increase in steam-producing power when the boilers were being fed from the Thermal Storage vessels was 22 per cent., which was taken off the top of the curve; that was to say, the work stored in the bottom cross-hatched areas was given out on the peak of the load. If anyone wanted to say that was incorrect, he would not be wrong, because the heat curve was given taking the varying feed temperature into account. The feed temperature was $150^{\circ} \mathrm{F}$. with 
(Mr. Druitt Halpio.)

a large volume of water, and also a large volume of gas going through the economisers, and it was higher at light load, coming up to as much as $220^{\circ} \mathrm{F}$.

Fig. 2, Plate 15, showed the combined general arrangement of one of the existing boilers and the Thermal Storage vessels now being fitted, two to each boiler." The existing boilers were marked A, and the boundary line below the top girders which carried the condensing tanks limited the available room. In the remaining space the vessels were placed. The only alteration that was made was that a hole was cut in the top of the boiler shell, a saddle piece riveted on, and a pipe put up which acted as a support to the ressel and had a connection for the steam and feed-water into the boiler, so that the pressure and the temperature in the Thermal Storage vessels and in the boiler drums were always constant. The Thermal Storage vessel was only rigidly connected at one point, so that it was perfectly free to expand in all directions, being carried at the other end by a suitable cast-iron supporting saddle-piece. The only other change that was made was in the feedpipes, which were disconnected from the boiler steam drums and carried up into the Thermal Storage vessel, and the whole of the feed passed direct into the vessels, and then passed through tho feed regulating cock and pipe into the boiler, as shown on the diagram. Supposing in the morning with a light load the water was down to the lowest water-level in the Thermal Storage vessel: if 10,000 lbs. an hour was being used in the engines, 20,000 lbs. would be pumped in; 10,000 went into the engines and the remaining 10,000 gradually accumulated until 3 or 4 o'clock, when the heavy load came on, so that when the ressel was full there was a charge of 20,000 lbs. available in each vessel at the same temperatures and pressures as existed in the boilers; thus at the top of the load, instead of having to evaporate from $150^{\circ} \mathrm{F}$., they were only putting in the latent heat, as the temperature of the feed from the vessels was already $388^{\circ} \mathrm{F}$., equal to the temperature in the boiler. In order to give some idea of what that meant, he might say, that as $20,350 \mathrm{lbs}$. were stored in each vessel, and 4,843,300 British thermal units were therefore contained in each vessel (this amount 
being the product of the available amount of feed-water into the difference between the temperature at which the feed was received at top load, viz., $150^{\circ} \mathrm{F}$. and the temperature at which it entered the boiler, viz., $388^{\circ}$ F.), the total working pressure was $215 \mathrm{lbs}$. per square inch, this increase temperature being, of course, $238^{\circ} \mathrm{F}$., and there were ten of those vessels. The steam used for the engines in that particular station was $23 \mathrm{lbs}$. per kw.-hour.

Each of the ten vessels stored up $250 \mathrm{kw}$.-hours, which gave 2,500 kw.-hours to help them over the top of the losd. The reason that system was adopted in connection with the new plant was very simple. The engineer went very closely into the question of the figures, and obtained tenders for the boilers and buildings, and without making any allowance for the value of land, which was very high in London, it was found that Thermal Storage only cost 70 per cent. of the price of new boilers without contingencies. In connection with the question of price, Mr. Miller was good enough to give him the figures that actual electriç- storagō batteries power had cost him. Four batteries were put in of 1,200 kw.-hours, which, without buildings, cost $£ 7,500$, so that the electric battery power cost exactly $6 \frac{1}{4}$ times as much as the Storage. Another point in connection with the battery was what would be got out of it. Fortunately on that head he was ablo to give very exact figures. In one of the Thermal Storage vessels Messrs. Willans and Robinson had put up at their Rugby Works they kept the vessel filled with steam for eleven hours. Thoy put a thermometer in the top, in the middle, and in the bottom of the veseel, and calibrated them carefully with a very accurate steam-gauge. They worked with the thermometer to see what result was boing obtained, the final planimetered that in the test the drop in the temperature of the feed-water entering the boiler was $3^{\circ} \mathrm{F}$. below the steam temperature. A drop of $3^{\circ} \mathrm{F}$. represented a loss of 0.8 of 1 per cent. He did not know whether any of the electric accumulator people would get a less loss than 25 per cent. in watts delivered.

Referring to what the actual losses by radiation were on the vessels, the results obtained at Rugby on a vessel 4 feet in diameter by 16 feet 8 inches long, showed that they were condensing 
(Mr. Druitt Halpin.)

something like 24 lbs. or 25 lbs. of steam per hour, representing $3.2 \mathrm{lbs}$. of cosl. In another vessel 6 feet in diameter by 19 feet long, in another place, the measured condensation over ten hours was $1.125 \mathrm{lbs}$. of coal per hour. That showed that at Rugby $1 \mathrm{lb}$. of coal was sufficient to deal with the radiation from 73 square feet of heating surface, whereas in the other case $1 \mathrm{lb}$. of coal would deal with the radiation of 231 feet of radiating surface, a proportion of 3 to 1 merely in the efficiency of the two different non-conducting materials. Messrs. Willans and Robinson used a most beautiful and costly material, but unfortunately it had not the same effect. He could give some other figures which might be of interest. Last April he tested with an assistant a plant of tho old type, which had boen at work for ten or twelve years. For one day they ran nine-and-half hours without storage, and the next day ten hours with storage. The water was very carefully measured by a Schönheyder watermeter, which was calibrated before and after the test; the coal was also weighed, its value on both days being tested by a calorimeter, so that they knew exactly what they were doing. The result was an increase of evaporative efficiency of $21 \cdot 7$, when Storage was being used, without giving credit for the difference in the thermal value of the cosl.

A test much more interesting to the meeting would be the last one he would give, which took place practically without his knowledge; the tests were finished before he knew they were being made, his clients determining to test the apparatus for themselves. They ran from the 10th May last to the 7th June, day and night, Sundays included, with varying loads, which were very light during aight hours of the night, carefully mensuring the whole of the coal snd water. The result was that $334,560 \mathrm{lbs}$. of cosl were burned, and 2,786,720 lbs. of water evaporated, showing that with exceedingly low class Midland slack, costing 6s. $6 d$. per ton delivered in the bunkers in Birmingham, an evaporation of $8 \cdot 12$ was being done from and at $212^{\circ} \mathrm{F}$. They then ran from the 21 st $J$ une to the 19th July under the same conditions, evaporating 2,223,270 lbs. of water, and burning 352,464 lbs: of coal, and in that case the evaporation, from and at $212^{\circ} \mathrm{F}$. as before, was $6 \cdot 7$. That test 
confirmed the figures he obtained in his own test, his figure for two months being $21 \cdot 7$, while his clients' was $21 \cdot 35$. In addition to the advantage of an increase of economy during the whole time of evaporation, there was a further adrantage in getting the greater part of the dirt and lime in the feed-water deposited in the $\Lambda^{\text {ressel, }}$ and in some cases, the whole of the dirt in the feed-water was precipitated; whereas, if they passel the feed-water through an economiser in order to get the bonefit of the incrensed temperature, the lime in the water was depositel inside the pipes, where, owing to the relatively high temperature outside, it was baked on in a very hard condition, and was very difficult to remove.

On page 23 of Mr. Stromeyer's roport for 1902 to the Manchester Steam Users' Association, he referred very fully to the undesirable amount of heat storel up in the brickwork: of water-tube boilers, which became most inconveniently evident when the boilers had suddenly to be taken off the load. In this case, in order to get rid of this accumulated heat, it was necessary to continue pumping in feedwater, and allow the steam produced to blow off through the anfety valves. Where Thermal Storage was used this waste did not occur, as the heat remaining in the brickwork was all usefully applied in raising the temperature of the feed-water pumped into the Thermal Storage vessels for use during the time the next heavy load came on.

The apparatus had also been applied to locomotives, the boiler being fed from the Thermal Stornge vessel when going up hill, the vessel having been previously filled while the engine was ranning down bill. By this means an increase in boiler steaming power was produced amounting to 30 per cent.

Mr. Gronge Wrikinson thought the Paper was full of interest to all engineers connected with the generation of power; it simply bristled with points upon which profitable and interesting discussion might be raised. The one statement which impressed him most in reading the Paper was that on page 512, namely, that, taking the average results from the tabulated statements in 1902 , condensing stations were not, by some considarable extent, so efficient, in point of works costs and fuel costs, as non-condensing 
(Mr. George Wilkinson.)

stations. Having read that, he looked through the Paper to find out if the author gave any reasons for such a startling conclusion. He found one (page 521) under the heading "The Station Engineer," where the author said "One explanation of this"-i.e., the economy of non-condensing plants- "may be that less plant requires less looking after and less repairs ; thore is also less capital invested." The capital in rested did not affect the works costs, but the other did. The author continued, "But a well-managed condensing station with economical engines, fitted with their own condensers, \&c., is certainly the most economical type of station." He had emphasized the "well-managed" because he thought that probably condensing stations, taking them all round, were as well-managed as noncondensing stations. He was thoroughly in accord with the author when he advocated the use of complete units, such as he described (page 516). The author pointed out there a general instance of a station which was fitted with a separate condensing plant common to the whole of the generators in the station, and it was not surprising to him, after having seen a number of such stations, that the economy came out so low; because when one came to consider that the range of output of those stations, especially lighting stations, varied from 100 per cent. for a short time down to 10 per cent. for a very much longer period, and that in such stations one often found the condensing plant engine of larger size than the day-load engine, and of a less economical type, it was a fertile source of loss; whereas if each generating unit were fittod with its own condenser, and made to drive its own air and circulating pumps, very much better results might be obtained.

He would like to ask the author whether he could give more in detail the black sheep amongst the condensing stations which lowered the averages so much that they compared unfavourably with non-condensing plants. The author mentioned (page 521) a fertile source of loss in station plants, namely, the capacious appetite of the boiler feed-pumps for steam. That was a loss which was common to both non-condensing and condensing stations, but he did not hesitate to sny that in stations where the output varied very considerably, as it did in most electricity works, feed-pumps were altogether a 
mistake for boiler-feeding purposes. He had designed and successfully introduced into several electric-supply stations in the country a new arrangement of injectors for this purpose, shortly described as follows:-Injectors in various sizes (so that a wide range of feed could be obtained" were "banked" in a row; the bank was divided in the contre by valves inserted in the steam, water, and delivery pipes. The steam to the injectors was taken in at each end of the bank, thus providing a duplicate service; a dual water-supply was also furnished by connecting at each end of the bank, and in like mannes duplicate deliveries to the boilers were provided. Instead of using live steam from the steam ring, all the water separators and all the steam-jackets of the engine cylinders were connected to a small sterm-pipe, which in its turn fed the bank of injectors. By this method, although the duty of each individual injector was curtailed, the enormous advantage was obtained of doing away with steam-traps; and the injectors were able to pass the whole of the condensed water from the separators and cylinder jackets through again into the boilers. It was found also that the injectors were quite capable of taking hot water from any ordinary hot-well, providing the injectors had no high lift on the suction. This arrangement was simple, efficient, and very economical, as proved by a number of years of continuous working.

The author drew certain conclusions (page 522) between steamturbines and reciprocating plant, and he rather joined issue with him on that matter. The Newcastle station was quoted as being the most economical steam-turbine station, but that came out rather at a disadvantage when compared with the South Shields station mentioned also in the same paragraph. He thought it was only fair to eay that many of the steam-turbines in the Newcastle station. were of an old type; much of the pioneer work had been done there, and their economy could not be equal in any sense to the present-day turbines. Another point was that South Shields, which had triple-condensing engines, had a load factor of $14 \cdot 6$ per cent., whereas the Newcastle station had a load factor of only 13.67 fer cent. Of the other steam-turbine stations mentioned, Scarborough had a load factor as low as 9.81 per cent., Cambridge only 8.59 per 
(Mr. George Wilkinson.)

cent., and Melton Mowbray only $6 \cdot 16$ per cent. In connection with this matter he had a few months ago the adrantage of testing a $300-\mathrm{kw}$. triple condensing engine, direct-coupled to a generator, and a $300-\mathrm{kw}$. turbo-generator working under precisely the same conditions, excepting that in the case of the vertical triple-expansion ongine the superheat was somewhat greater than in the turbine, but in the turbine test the vacuum was a little better. At full load the vertical triple engine came out at $23 \cdot 9$ lbs. per $\mathrm{kw}$. generated against the turbine $22 \cdot 76$; at half-losd the vertical triple engine came out at $29 \cdot 4$ against the turbine $24 \cdot 1$. He did not mention these figures in any spirit of cavil, but to show that there was little difference between a modern steam-turbine plant and a high-class tripleexpansion reciprocating engine plant in point of steam consumption.

Likewise in connection with gas-engine stations, the author quoted Leigh against Northwich. Leigh had a load factor of 12.32 per cent., and also had the advantage of being on a coal-field where coal was cheap, whereas Northwich had a load factor of only $9 \cdot 34$ per cent., and purchased their gas from the Brunner-Mond $\mathrm{Co}$, which company, no doubt, made a profit on the transaction. With regard to losses in mains, the author allowed 20 per cent. (page 511). He thought ho had erred on the safe side there, because at any rate in alternating systems 30 per cent. or 33 per cent. would be much nearer the mark.

Mr. Charles Forgan thought the Paper would have been of more value to central station engineers, if the author had investigated his figures before publishing them, not merely taking them from the "Electrical Timss," because he did not think they went to the point. For instance, the author stated (page 514) that the works costs of the three railway power-stations were heavy. He would like to know whether the other power-station results were obtained on a similar basis as the railway generating stations. Being connected with one of those railways, the Central London, he thought the figures were very misleading; more details might have been given. For instance, on page 536 the Central London Railway's works costs per unit was given as $0.92 d$. That included power-house costs, the wages of 
locomotive drivers, costs of repairs to locomotives, and the wages of the men employed in that department. The total cost which the $0.92 d$. referred to was $£ 31,537$ for the half-year. The power-house costs were $£ 18,049$, while $£ 13,488$ represented the cost of the locomotives and car department. Taking the power-bouse costs and works costs alone, the figure obtained was just a little over $0.5 d$. per unit generated, that was taking the wages of the office staff, working expenses, water, gas, waste, store repairs and maintenance of the power station. He thought $1 \frac{1}{2}$ lbs. of coal per I.H.P. was a very good figure if it was maintained in most stations.

He thought the author was in the same boat as tho speaker referred to on the previous day in comparing badly equipped condensing stations with well-equipped non-condensing stations; if the author had taken modern well-equipped condensing stations, he would have found the comparisons come out better. Fur instence, the Central London Railway had not the best condensing plant, and the engineers very soon knew it in the matter of boiler-power if they had trouble with the condensers. With reference to the Scotch mist, there was no doubt that Lancashire boilers had good staying powers; and he thought it was an adrantage to power stations to have them in parallel with water-tube boilers, because the Lancashire boiler was very good for steady loads, bnt if one was in a hurry a watertube boiler was wanted. He had had a good experience with water-tube boilers at the London Electric Supply Corporation and the Central London Railway, and in an extension which had just been carried ont four Lancashire boilers bad been put in, and a saving resulted when the boilers were run with the water-tube boilers.

He thought it would have been more to the adrantage of station engineers had the author dealt more with the different costs of coal. The mechanical stoker had a good deal to do with the question. A lot of engineers thought the mechanical stoker a boon: he thought otherwise. At the Central London Railway two years ago they changed from using the mechanical stoker to hand-firing-he would not mention the particular type-because he did not think there was much to choose between them. Difficulty was experionced in getting 
(Mr. Charles Forgan.)

the men to do exactly as one wanted, but after six months' running the coal costs were $£ 4,000$ a ycar less than they were previously with mechanical stokers. He could not say that that saving was all due to the stokers, because he had not much to do with the company while the mechanical stokers were in use; but, under instructions from his chief, he made the change he had referred to. The units generated were practically the same in both cases, while the engines had only undergone the usual overhnul and adjustment; therefore the stoking had a good deal to do with the saving in fuel.

Mr. J. D. Barure thought the author was not quite fair to station engineers in general in regard to condensing. They were not blind to the advantages of a good vacuum, and the case cited (page 516) of 23 inches at the condenser and 11 inches at the engine exhaust was quite an exception. Driving from the main engine had one disadvuntage, in that if onything went wrong with the air and circulating pumps it disabled the main engine temporarily, until the pumps were disconnected. Probably, in general, the best method for central-station working would be to drivo the pumps by motors, and to have intercommunication pipes and valves between the condensers, so that, in the event of any hitch occurring on the one condenser, a rensonably good racuum could be obtained by coupling on to another one. He did not think that the last sentence of the first paragraph on page 517 was quite correct, for the author stated that the steam required by the auxiliary machinery was altogether saved. That, of course, was not so; for the air-pumps and the circulating pumps had work to do in moving the water against a certain head, which entailed an expenditure of: power and steam, whether by the main engine or by an auxiliary engine.

The author quoted (page 518) a test of a 120-H.P. triple-expansion condensing engine, the exceptionally good result of $12.54 \mathrm{lbs}$. of steam por I.H.P. per hour being obtained, with a boiler pressure, of 157 lbs. per square inch, and no suporheat. The mechanical, efficiency of this engine, including the driving of all the pumps and the brake-wheel friction, was given as $93 \cdot 5$ per cent. This certainly 
seemed a high mechanical efficiency for a triple-expansion engine, and $12.54 \mathrm{lbs}$. per I.H.P. was an exceedingly low steam consumption under the conditions stated. Working these figures out they eame to $\mathbf{1 3 . 4}$ lbs. per B.H.P., and, if coupled to a dynamo with only 90 per cent. efficiency, which was low for dymamo, an electrical H.P. consumption of about $15 \mathrm{lbs}$. would be obtained, or a consumption per kilowatt of say $20 \mathrm{lbs}$. With all respect, he did not think the author's firm would care to guarantee a consumption of 20 lbs. per kilowatt per hour under those conditions.

Mr. MoLares interjected that he had given the names of the men who had carried out the tests.

Mr. Batury said he would like to know, in connection with these tests, what the vacuum and the temperature of the steam were. It did not state the amount of superheat, if any, and what allowances were made for leakage, etc.

Mr. MoLarex replied that there was no superheat.

Mr. BaILIE, continuing, said that the author advocated running the auxiliary engines non-condensing and using the exhaust steam for heating the feed. Why not combine the two, by runaing both the main engines and the auxiliary engines condensing; and, ag was done at West Bromwich, pass the exhaust steam through feed. heating tabes first and then through the condenser, imparting say $60^{\circ} \mathrm{F}$. to the feed, and maintaining a vacuum of sey 26 inches on the condensers ? He thought perhaps the greatest economy was obtained in that way.

The anthor reforred in a somewhat disparaging manner to steamturbine stations. It was rather a pity he had done so, unless, as he said, it was for the purpose of provoking discussion, because it was quite unfair to compare an old station like the Newcastle and District with a practically new station such as South Shields. The Newcastle and District station was partly condensing; the South Shields 
(Mr. J, D. Bailie.)

station was wholly condensing. The Newcastle and District station had to pay for the carting of coal and also for the carting of ashes, whereas in the South Shiolds station tho coal was practically taken from the boats to the furnaces. The heading "Fuel Costs" was quite mislesding, in that it did not take into account the different itoms which were included. The author also gave the" stations mentioned by him as the only four stations wholly equipped with turbines, but of course there were others, including West Bromwich, Shipley, etc. Three of the four cited, namely, Newcastle, Cambridge, and Scarborough, were the oldest. The fourth, Melton Mowbray, was a very small station, with a total capacity of only 150 kilowatts, so could nut fairly be compared with a large station. For comparison with turbine stations, the author selected South Shields, a comparatively new station with every facility for economical working, but he omitted to take a station such as Oxford. At Cambridge the total cost of fuel per unit sold was $0.66 d$., whilst at Oxford it was $0.74 d$. The stations were started in the same year, and the conditions as to irregularity of load were probably much alike, both being University towns, yet the cost of coal, the total works cost, and everything else came out higher at Oxford than at Cambridge. A comparison between Cambridge and Oxford would have been fairer than one between Cambridge and South Shields, though probably the anthor would dispute this, and would say that the reason for the higher fuel cost at Oxford was the system of distribution in vogue; if so, his whole Paper fell to the ground at once, for it showed the fallacy of abstracting isolated columns from tables such as those published in the "Electrical "Times," and assuming the figures in them to represent the economy of the engines. Melton Mowbray was, as previously stated, a very small station, with $150 \mathrm{kw}$. capacity and an output of 43,000 odd units per annum, as against $1,600 \mathrm{kw}$. capacity and a million odd units ontput at South Shields.

After reading the author's Paper, he wrote to the Melton Mowbray engineer, who informed him in reply that, since the addition of a larger turbo, the cost of coal had been reduced to $0 \cdot 388 d$. per unit sold, and that it was coming down every week. 
He also wrote to the engineer of West Bromwich, who had very kindly furnished him with a copy of their official returns as prepared for the Board of Trade. The total works cost there, although it was comparatively a small station, came out at $0.65 d$. per unit sold, and the cost of fuel at $0.35 d$. - they were below that at the present time. That was a -better: result by far than South Shields, considering the difference in size of the stations, etc.

In regard to the question of superheat, he presumed the suthor's remarks referrod to separately fired superheaters. If they referred to flue superheaters, he did not agree with him; there was very little upkeep or attention required with the flue type of superheater, except in cleaning out the soot deposited from time to time-a simple matter. With superheaters of the ordinary Babcook and Wilcox type, about $1,500^{\circ}$ F. superheat was regularly maintained at a number of stations which he knew-West Bromwich, etc. The economy of superheat to that extent was about 15 per cent. of the steam consumption, so that he could not quite that superheaters were a disadvantage in a station.

Mr. T. L. Muler thought the point in the Paper which probably interested engineers most was that referring to the superior economy of the non-condensing electric lighting stations over the condensing. He did not think, however, that if all the facte bearing upon the question were taken into consideration, the difference shown would be found to be due to whether the engine were non-condensing or condensing. In the remarks he made after the reading of the Paper, the author, he thought, gave one reason for the figures, and Mr. Wilkinson in his remarks gave another which he proposed to deal with very shortly. Dealing first with the relative economy of non-condensing and condensing engines, it was a well-known fact that working under similar conditions the condensing engine, at all loads, gave a greater economy in water consumption per horse-power hour than the non-condensing engine. Provided, then, that the conditions of loading were the same in the non-condensing and condensing stations, it appeared to him that there could be no 
(Mr. T. L. Miller.)

increased economy of the non-condensing engine over the condensing. This being 80, it was necessary to look in some other direction for an explanation of the very startling figures given by the author. Now, in addition to the type of plant employed, the effciency of a generating station, stated in terms of cost of coal per Board of Trade unit sold, depended, amongst other things, upon :-

1. The system of supply.

2. The "load factor."

3. The cost of fuel.

4. The personal factor in the management.

All these factors had a considerable bearing upon the costs of production. Dealing with the first factor, namely, the system of supply, it might be pointed out that the stations in this country could be divided roughly into two clusses, one the higin-tension and the other the low-tension stations. The high-tension stations, for the most part, were put down in districts where the demand was sparse, when one had to go for considerable distances in order to get consumers. On the other hand, low-tension stations were put down in more densely populated districts. Leeds was an example to the contrary, and there were other such exceptions throughout the country. In the high-tension stations the difference between the units sold and the units generated was very much greater than in the low-tension stations ; for example, from an examination of the records of fourteen low-tension stations and twenty high-tension stations, he (the speaker) found that the average percentage of units sold to units generated was $86 \cdot 6$ in the low tension, and but $67 \cdot 3$ in the hightension stations. Allowing for these losses, it might reasonably be expected that the coal costs in the: high-tension stations would be higher than those in the low-tension stations. In addition to this, however, the low-tension stations bad the advantage of batteries, which, in the smaller stations, tended to further reduce the costs; as well as a considerable motor load during the day, giving an increased "load factor." Bearing in mind these facts, and dividing the stations into two classes, high and low tension, it would be seen what a great effect the system of supply had on the 
costs, and how fully their ideas of the economy, in terms of coal consumption per unit sold, of the condensing engine over the non-condensing were borne out. Leaving out of consideration the Metropolitan Company's stations, part of which were condensing and part non-condensing, and also the eight stations where steam was partly supplied by refuse destructors, the stations might be grouped as follows :-

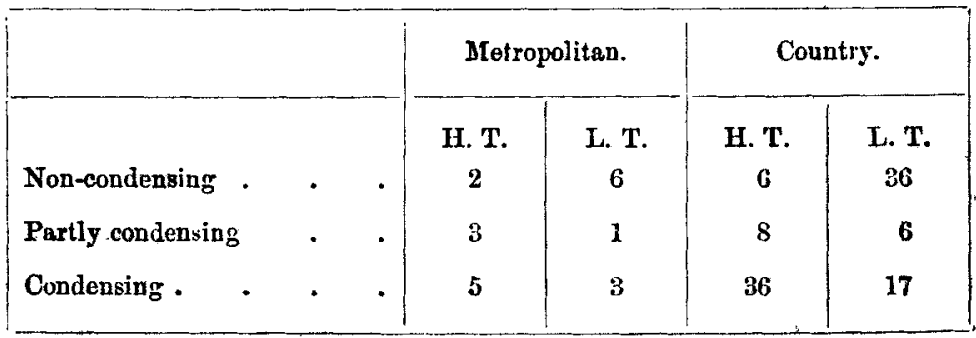

It would be noticed from these figures that in the non-condensing stations the iow-tension systems of supply were greatly in excess of the high-tension, and from the data already given regarding the better efficiency of the low-tension systems, it would be readily understood that the non-condensing results would show a high average efficiency. In the condensing stations, on the contrary, the high-tension systems of supply were in excess of the low-tension; and as a consequence a lower average effieienoy wonld be obtsined for the condensing stations. This disproportion between the high and low-tension systems of supply in the non-condensing and condensing stations appenred to him to account largely for the supposed superior economy of the non-condensing stations referred to by the author.

Dealing, then, with the euthor's figures of cosl costs per unit sold, and omitting as before the figures for the Metropolitan stations, and the eight "destructor" stations; he (the speaker) subdivided the purely "lighting" stations into two groups from which he obtained the following figures for the average cosl costs per unit sold :- 
(Mr. T. L. Miller.)

\begin{tabular}{|c|c|c|c|c|c|c|}
\hline & & & \multicolumn{2}{|c|}{ Metropolitan. } & \multicolumn{2}{|c|}{ Country. } \\
\hline & & & H. T. & L. T. & H. T. & L. T. \\
\hline Non-condensing & - & . & $1 \cdot 17$ & 0.93 & $1 \cdot 72$ & 0.63 \\
\hline${ }^{7}$ Partly condensing & & $\because$ & $* 1 \cdot 14$ & $0 \cdot 85$ & $1 \cdot 03$ & $0 \cdot 54$ \\
\hline Condensing . & - & . & $1 \cdot 0 t$ & $0 \cdot 95$ & 0.90 & $0 \cdot 61$ \\
\hline
\end{tabular}

Referring to the high-tension stations, he pointed out that in both the metropolitan and country stations the condensing stations showed a greater economy than either the partly condensing or noncondensing, and that the partly condensing stations in like manner showed a bettex economy than the non-condensing. In the lowtension stations, however, the coal costs in both non-condensing and condensing stations were sensibly the same, while the partlycondensing stations appeared to better advantage than either the condensing or non-condensing stations. This difference in the metropolitan low-tension stations, he thought, might be accounted for by the better load factor of the non-condensing stations, which, according to the "Electrical Times," varied from 11.38 per cent. to $22 \cdot 74$ per cent. in the non-condensing stations, was 18.6 per cent. in the case of the partly condensing, and varied from $7 \cdot 87$ per cent. to 16.55 per cent. in the case of the condensing stations. In the country stations again, the load factor and the price of coal would very largely affect the results, some stations being in the neighbourhood of the coal-fields, and having good motor loads, while others had to pay a heary price for coal and at the same time had practically no day-load. In addition to these points the personal factor in management had a very considerable influence on the reduction of costs, and while he (the speaker) thought that the system of supply had a great influence on the question of costs, partieularly in the smaller towns, he was fully aware that it could not account for all the variations noted. The "policy" of putting condensing plants down in purely lighting stations was not under discussion, and he did not therefore touch on it. 
The next point to which he wished to refer was with regard to the driving of auxiliaries. The author had given some very high figures of steam consumption for driving auxiliaries, but he thought that in most modern stations the auxiliaries, and especially the air and circulating pumps, would be electrically driven. In that connection he would refer to a Paper read before the Manchester Section of the Institution of Electrical Engineers* by Messrs. Taite and Downe, in which the results of the running of the plants at the Salford and Southport stations were given. In those stations the auxiliaries were electrically driven and the energy metered. Regarding the metered anits, it was found that the condensing plants taken throughout the year accounted for 4.67 per cent. of the total units generated, and the boiler feed-pumps $1 \cdot 18$ per cent. The electrically driven plant cost about three and a half times the steam plant; but even allowing for the increased capital cost there was atill a considerable seving in electrical driving. Mr. Druitt Halpin earlier in the discussion gave some figures with regard to gas-driven plants; he (the speaker) would like to give some figures with regard to another gag-driven plant, a 500-H.P., in which Mond Gas was used, withont ammonia recovery. The load factor of the plant was 55 per cent., and the cost of fuel 118. 8d. per ton. Under these conditions the costs were as follows :-

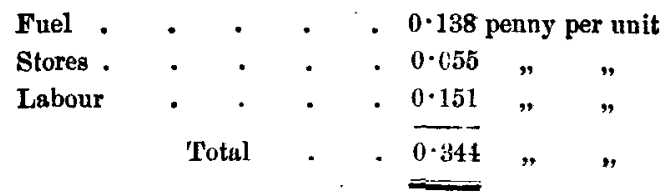

The only other point to which he would refer was the question of the gas-driven stations. Attention had alroady been drawn to the fact that the Northwich station, referred to by the author, bought its gas from Messrs. Brunner Mond and Co., the price paid being 2d. per 1,000 cubic feet. The author also gave some figures for another gas station, Redditch, where the fuel costs were $1 \cdot 3 d$. per unit. The most recent gas station was that at Walthamstow, where Dowson gasproducers and anthracite coal were used. From the return of the past

* Journal 1903, vol. 32, page 1050. 
(Mr. T. L. Miller.)

year's running, which had just been issued, he found that the fuel costs at Walthamstow amounted to $0.42 d$. per unit, and tho works costs to $1 \cdot 18 d$. per unit, figures that were very much below those given for Redditch.

Mr. Mark Robinson, Member of Council, thought the members were very much indebted to the author for deducing so many instruetive and valuable conclusions from statistics which indeed were always before them, but which without such guidance they too often found dry and uninteresting. No doubt what would attract attention chiefly were the remarks upon the comparative economy of condensing and non-condensing engines-a very important subject, to which, in his opinion, not nearly sufficient attention had been given by engineers. He could not help thinking that the last speaker had chiefly been showing how figures might be made to prove anything, because there was nothing to be surprised at in the fact that the non-condensing lighting stations gave the best results. The old idea of a non-condensing engine was that it was a sort of poor relation, a sort of deformed example, of the only proper kind of steam-engine, the condensing engine-a view which might have been reasonable when all steam-engines worked at very low pressures. If one cut off the part of the indicator diagram which was below the atmospheric line, in a case where the initial pressure was not very far above that line, there was of course very little diagram left, but when one worked with high pressure, the part of the diagram above the atmospheric line was very much the largest part. In order to retain the smaller part, below the line, one had to spend much money upon condensing plant, and if the engine ran only for a few hours in the week, it was quite natural that it did not pay to do so. And not only would they have to pay interest upon the cost of the condensing plant, but the engine itself must be enlarged. It was sometimes thought that if one had a non-condensing engine and then added a condenser to it, more power should be obtained from it. That was a wrong idea altogether; the use of condensing, that is of the artificial removal of atmospheric back-pressure, was to enable expansion to be carried 
further, but for that purpose the cylinder volume must be increased to correspond. The non-condensing engine therefore was much cheaper and smallex than a condensing engine giving the same power, altogether apart from the outlay on air-pumps. When these considerations: were taken into account, it was not at all surprising that in stations not running with good load curves the noncondensing plants came out the best. In a power station, with a fairly even lood, of course condensing paid, but for almost any other class of station it appeared to him that the system of partial condensation was likely to be the best. When, on the normal average load, condensation could be used, the occasional higher load, the peak of the curve, would be best met by starting an extra noncondensing engine or engines.

It was with satisfaction that he noticed in Mr. MeLaren's tribute to the non-oondensing engine, what was really a recognition of the work of another Leeds man, the late Mr. Willans, who nearly 20 years ago made non-condensing ongines which used less than $18 \frac{1}{2}$ lbs. of steam por I.H.P., the same engines asing about $12 \frac{1}{2}$ lbs. when working condonsing. Between those figures there was no doubt a great difference, but in an engine which ran only intermittently, and for a very short time in each day, it quite possibly might not be worth while to save it. The author had endeavoured to account for the comparative non-success of the London condensing stations by speaking of them as pioneer stations, but he did not think that was correct, except with regard to the big station at Deptford, which fortunately was a law unto itself, and exceptionul. The old pioneer plants of the London stations had long since been done away with; the real pioneer stations in London were the non-condensing stations, such as those of the St. James's and Pall Mall, and of the Westminster Companies ; those stations used Willans non-condensing high-speed ongines, and gave excellent results. Only one power station, the great tramway station at Glasgow, seemed to do better in works costs per unit than the best of the lighting stations, and then it was only by the tiniest decimal, the figures for Glasgow being $\theta \cdot 6 d$. against $0 \cdot 62 d$. for Leeds and $0.65 d$. for Edinburgh. He did not know the comparative cost of 
(Mr. Mark Robinson.)

coal in Edinburgh and Leeds, but he imagined Leeds had the advantage in that respect, perhaps suficient to alter the order in which they stood. It was very remarkable that Edinburgh, which took this high position in the entire list, was another example of a noncomdensing station. In view of this he felt justified in entering a protest against the author's condemnation (page 519), where ho mentioned " uneconomical three-crank compound, tandem, condensing engines largely used in lighting stations," beceuse these words seemed to refer to the very engines, the Willans high-speed three-erank engines, which, even without the advantage of condonsation, gave such splendid results at Edinburgh. Further, in connection with the London stations, it would be found in Table 3 (page 525) that the Kensington condensing station, which had those very three-crank engines, tied with another London condensing station for the second place on the list, and was only just beaten by one other station, which had engines of another kind.

Mr. MoLaren, interposing, stated that he specially mentioned that the most economical stations were fitted with non-condensing engines.

Mr. Mank Robinson, continuing, said that piston-valves were somewhat strongly condemned in the Paper. He could say, from an experience of many years, based upon the use of many hundreds of Willans engines fitted with piston-valves, that these valves, when properly made (which was often not the case) remained tight and efficient. His firm had the strongest reason for discovering the best valve, and their continued faith in the piston-valve was simply the outcome of long and oft-repeated experiments; they could simply find no sort of valve which in actual nse was, and remained, so tight. The Edinburgh engines were, of course, piston-valve engines, and they had for the most part been running for years.

Mr. Michane Lonaridee, Member of Council, said the most striking fuct brought out by the Papor was the superior economy of non-condensing over condensing engines. One reason had been given by a previous speaker, but there was another which he thought 
must have suggested itself to many of the members, that was the exceeding badness of many of the condensing plants put down by electrical engineers. It was true that electrical engineers had taught steam engineers much, but it was equally true that they had much to learn themselves from the steam engineers. The surface of evaporative condensers, the capacities of air pumps, and the water cooling arrangements were often utterly inadequate, and the idea of the independent central condensing plant which seemed to find favour among electrical engineers was, when reduced to practice, generally a fraud. He strongly advised a separate condenser for every engine. The objection generally urged against this arrangement was the high speed of the engines, which made the driving of the air-pump difficult. But he would remind the members that there was a condenser which required no air-pump, and was therefore independent of the speed of the engine, a condenser which moreover occupied very little floor space, and that was the barometric 33-feet pipe condenser. The water could be supplied either by a centrifugal pump electrically driven, or by a small steam-pump such as the Worthington pump. If, however, a steam-pump was used, he thought it well to caution the members that the stop-valve for admitting the steam to the cylinder must be made exceedingly small. The ordinary stop-valve supplied with such pumps was well adapted for starting the run when the water had to be lifted to the top of the condenser; but the moment the vacuum was established, and the steam-pressure in the pump had only to overcome the friction of the water in the rising main, the quantity of steam taken by the pump became so small that the ordinary stop-valve had only to be opened a fraction of a turn, and he had found that when working in this way the vibration of the pump was very liable to shut the valve and stop the action of the condenser.

Professor Annolp Lupton said that he had several barometrical condensers working successfully. There could be no doubt at all, as stated by Mr. Mark Robinson (page 557), that in considering the kind of engine required for a central power station it was necessary to take into account the load factor. One kind of engine was very 
(Professor Arnold Lupton.)

suitable for 5 per cent. Ioad factor, and another kind more suitable for 50 per cent. load factor. Most of the stations were electriclighting stations. But in future they would have to deal more with electric power distributing stations. He had had some share in getting passed through Parliament two Acts providing for two large electric power generating stations involving two millions of capital each, so that naturally he had given a good deal of consideration to the question. He quite agreed with the author's remark that it was rather absurd to think that one could get an engine, turn its power into electricity, take it ten miles and drive a factory, and compete with another factory which bought its fuel as cheap and could buy an equally good engine. He and his colleagues never proposed such a thing. They thought they might supersede existing steam-engines of a bad type, using from 5 to $10 \mathrm{lbs}$. or even $20 \mathrm{lbs}$. of fuel per horse-power. Where extensions of power were required, and it was not convenient to put down a big engine, then electrical transmission would come in useful. There were economies. He had mado a few calculations to show what could be done theoretically. Taking the cost of fuel at a central generating plant distributing power over, say, a radius of ten miles, and taking the cost of fuel in a town for one of the bad engines, which someone said was iniquitous, he found that $£ 1$ spent in fuel at an electrical power central generating station would do as much work upon the material that had to be worked upon at the factory as $\$ 10$ spent on fuel at the factory. Taking the cost of 48 a ton at tho central generating station near the coal mines-which he knew to be a reasonable price in some districts-and taking the cost at $10 \mathrm{~s}$. a ton in the town at the small mill of 20 or 30 H.P, at the generating station, with every known device for economy, the I.H.P. would require .only $1 \frac{1}{2} \mathrm{lbs}$. of fuel per hour; and at the suggested small factory with second-class engine, it would require $6 \mathrm{lbs}$, thus making a ratio of $4 \times 2 \frac{1}{2}=10$ to 1 in fuel cost. In the electrically-driven factory the motor would be on the machine or on the countershaft, and in each case the power actually utilised in the machine was taken at one-half of the I.H.P. This calculation took no notice of any costs but the fuel cost. 
Mr. W. Price Aberi thought that not the least important part of Mr. McLaren's Paper was the pertinent diagnosis of the work done at central stations, enabling him to point out with such clearness the weak spots, some of which were a surprise to engineers-namely, that non-condensing plants on an average acted more economically than condensing plants. He (the speaker) could not pass over Mr. Forgan's condemnation of mechanical stokers;-some certainly friled, but the recognised good makes of mechanical stokers got over the three great causes of loss in handfiring in steam generating, namely, first, the entrance of cold air whilst charging the furnace by hand; secondly, the entrance of cold air whilst cleaning the bars; and, thirdly, the entrance of an excess of air though parts of the furnace charged too thinly, or where combustion had been'quicker than in the remaining parts. In most of the stokers used in the Leods electric light and power stations, members could see how the third loss was prevented by the utilization of a movable bar taking its own restricted air; so that in case of one part of the fire burning faster than another, it did not allow excess of air to pass through the thin part and rol the thicker layer of fuel of its allotted share. In several factories with which he had been connected, attention to these three points had reduced the fuel consumption very considerably, even in cases where expensively watched sciontific hand-firing had been practised, and much more so when the stoking had been done with officient mechanical stokers.

Mr. Alfred Saxon said there was one aspect of the tripleexpansion condensing engine which had not been referred to, and which, he thought, would account for its apparent loss of economy, namely, that in ashort run a triple-expansion engine would not be seen at its best advantage nor even at light loads. A tripleexpansion engine of 1,400 I.H.P. made by his firm was tested in November last year. In the first test of three hours in the morning the steam consumption worked ont, with saturated steam, to $12 \cdot 6$ ibs. per I.H.P. In the afternoon run the steam consumption worized ont 
(Mr. Alfred Saxon.)

at $12 \cdot 21 \mathrm{lbs}$, with practically the same load.* The following day the tests were continued with almost identical results. That served to show that in a long run, up to a given point, the triple-expansion engine became more efficient the longer it ran. The author had asked for some cotton mill engineer to give the cost of running a cotton mill for a week. A cotton mill in Lancashire, with an engine developing 1,000 H.P. could be run for $£ 20$ worth of coal per week of fifty-six hours, including the heating of the rooms and the stand-by losses. Taking fifty working weeks to the year and $£ 20$ per week, this would equal $£ 1,000$, and thus an engine of 1,000 I.H.P. could run for fifty-six hours in the reek at a cost of $£ 1$ per I.H.P. for a year.

Mr. McLaren, in reply, said that Mr. Wilkinson asked for the names of the black sheep. He had only to look down the Table of the costs per unit to find them. In connection with auxiliary machinery Mr. Wilkinson mentioned his injectors. That was a very interesting system. He had seen the method at work at Harrogate, and had adopted it with great success, using the injectors to feed the boilers, and taking the steam for the injectors from all the stearn separators. Some engineers might think that injectors would not work with water in the steam supply, but they did; as a matter of fact they worked splendidly.

He was not at one with $\mathrm{Mr}$. Wilkinson with regard to the turbine and the triple engine. Mr. Wilkinson's triple engine was practically a compound engine, the cylinders being proportioned for a low steam-pressure-it was only 120 or 125 at the time of the test; he had now got up to 130 by squeezing the Boiler Insurance Co. The condenser coupled to the engine was of the ejector type, and only gave a very poor vacuum indeed, something about 15 inches. The turbine worked with a splendid vacuum. It was well known that

* Corroborating the figures given by the author, a similar new tripleexpansion cngine to the one described above was indicated by the Engine and Boiler Insurance Co., on 2nd April 1903, and showed an efficiency of 91 per cent. 
about $1 \frac{1}{2}$ per cent. in economy was gained for every extra inch of vacuum obtained; if one intended to compare engines with engines, this must be borne in mind. Mr. Forgan explained (page 547) how it was that the works costs on the Central London Railway were apparently high, through outside expenses, that were not included in other stations, being added to them. He, the anthor, specially mentioned in the Paper that that was a point which required explanation, and the explanation given was satisfactory. Mr. Bailie mentioned (page 548) that the Metropolitan was not all condensing. As he already explained, he took the figures from the Tables published by the "Electrical Times." Mr. Bailie also doubted the possibility of the 12.5 and the 93.5 per cent. efficiencies being correct. There was no need for doubt in regard to the 12.5 ; it was e sily obtained with good engines, as $\mathrm{Mr}$. Robinson and Mr. Saxon had explained; but the 93.5 per cent. certainly required explanation. The efficiency came out rather over 91 for engine and brake combined. The brake wheel was 10 feet in diamoter, and the trough-shaped rim was for water cooling. Their contract stated that they finished at the shaft-coupling. They had nothing to do with the alternator, so he demurred to driving this large brake and including its friction as engine losses. Mr. Wilson Hartnell settled the point; he sent for a motor, obtained a light strap, and drove the wheel uncoupled, in its own bearings without brake straps on, but with equivalent weight added so as to give it the same pressure on the bearings; it was rather surprising to see how long it took to get the meter pointer quite steady; he believed it was found that $2 \frac{1}{4}$ E.H.P. was required to run the wheel to proper speed. That was how the figures were got ont, and he knew no man whom he would sooner trust to make a brake test than Mr. Hartnell.

Mr. Bailie also made some remarks with regard to the turbines and engines. He took the Newcastle station because it was the best. He thought it was an error in the "Electrical Times" tables to say that the district station at Newcastle was partly condensing; it was a fully condensing station. The turbines were not antiquated and outof-date; and there wore some of the best and latest types of them in that statiun. There was very little difference between Newcastle and 
(Mr. McLaren.)

South Shields as regards corl supply. He believed that South Shields at that time was paying very much more for coals than Newcastle. Mr. Bailie also mentioned Oxford and Cambridge; but there was no parallel between those two towns. Oxford was an isolated case practically, in regard to the system of distribution. The current left the station at 4 lbs. of coal per kw.; and the members could easily reckon out from the Tables what it amounted to when it was distributed. The price of coal was much higher at Oxford than at Cambridge, Cambridge being on one of the main coal lines to London. Mr. Miller gave some interesting details in connection with the mains and their effect on the costs; but he would reply to his remarks more fully in writing (page 573).

There was one point he would like to mention in connection with the method of distribution of power by electric energy. Ten years ago in his works they took out their steam-engines and boiler used for driving the works and put in a new one, thereby saving over 60 per cent. in fuel. If there had been any electricity employed, all that saving would have been put down to the electricity, but luckily there was no electricity connected with it. It would thus be seen that a great saving could be made, by simply putting in an efficient steam-engine in place of a wasteful one.

\section{Communications.}

Mr. E. G. Constantise wrote that Mr. MuLaren was to be heartily congratulated on bringing before the Institution such an important subject as "Fuel Economy in Generating Stations" and the anomalies which existed in the generation of steam in this enormous and rapidly extending industry. It might $\operatorname{come}$ as a surprise to the author that, although, as he stated (page 520), "Engine-builders (may) find much less difficulty in getting their guarantee figures from the honest steam of the Lancashire or other cylindrical boiler than they do from 'the high-pressure 'Scotch mist' given off by the 
now fashionable box of water-pipes," an analysis of the figures supplied showed that the boxes of water-pipes gave better results in working than either Lancashire or other shell types of boilers. Of the more economical stations (lighting and traction) referred to in the Tables, 29 generated or sold electricity at a coal cost per unit of $0.5 d$. or under. Of these stations 10 were equipped exclusively with Lancashire boilers; 3 with shell boilers other than the Lancashire type, 10 with a mixture of water-tube and shell boilers, and 6 stations with water-tube boilers exclusively.

The average coal cost per unit sold or generated at the 29 stations worked out as bolow :10 stations with Larrashire boilers only . . . . 0.435 per unit. 3 " " shell boilers other than Lancashire . . 0.393 " " $10 "$ " mixed water-tube and shell boilers . . $0.390 "$ " 6 " "water-tube boilers only . . . . $0.385 "$ "

It would thus be seen that the coal cost per unit was highest in those stations in which only Lancashire boilers were employed, and that those exclusively equipped with water-tabe boilors generated their electricity at the lowest cost for fuel. In passing, it might be noted that in the very best example given in the Tables, that of Glasgow Electric Tramways, the cost of $0 \cdot 19 d$. per unit was obtained with the author's "boxes of water-pipes." Had the 10 stations fitted with Lancashire boilers been equipped with "boxes of water-pipes" working at the average cost for the 6 stations given in the Tables, the saving in cost of fuel would have amounted to over $£ 7,000$ per annum.

Carrying the comparison farther, it was interesting to observe that the works costs per unit generated were also the lowest in the stations furnished exclusively with water-tube boilers, and bighest in those having Lancashire boilers exclusively-the relative figures being $0.86 d$. in the water-tube boiler stations against $1.024 d$. in the Lancashire boiler stations. It might be contended that the better results shown by the water-tube boiler stations were due to cheaper fuel, more economical engines and better management, but the first reason was disposed of by the fact that of the Lancashire boiler stations six were actually on the coalfields, and four were within a 
(Mr. E. G. Constantine.)

distance of 30 miles of collieries producing steam-eoal; while of those stations equipped with water-tube boilers-one was in London, two were in Ireland, two in the Glasgow district, and only one on the coalfields. Doubtless economical engines and good management were responsible to some extent for the results, but these advantages were certainly not peculiar to exclusively water-tube boiler stations. The Lancashire boiler was a good old faithful servant, but where economy, space, and high pressures were in question it was not the best boiler, and must give place to the " box of water-pipes."

Although the "Scotch mist" box of water-pipes (illustrated in connection with Mr. Halpin's remarks, Plate 15) was by no means the most efficient water-tube boiler in use, even it has been proved more efficient than the Lancashire boiler; and one might almost be tempted to infer that, when once the engine-builders' guarantees were fulfilled, engines worked more economically on "Scotch mist" than on the honest steam referred to by the author.

Lt.-Culonel R. E. Crospton, C.B., wrote that Mr. McLaren dealt with mutters of supreme interest not only to electrical engineers but to all users of power. They were indebted to him for calling attention to certain matters which were far too much neglected by all users of steam power. He thoroughly agreed with his remarks on page 519 , in which the author attributed a very large share of the want of economy of steam-engines of all classes to valve and piston leakage, especially the former. He also agreed with him that huge losses occurred through leaky piston-valves, and that Corliss valves, plain slide-valves, and properly balanced flat slide-valves were much easier to keep steam-tight. The author's remarks on this matter must be read in connection with Callendar and Nicolson's valuable Paper* on "The Law of Condensation of Steam." Their Paper showed conclusively what a large proportion of the so-called condensation losses were really due to valve leakage losses and subsequent re-eraporation of the steam, and that this leakage was great when saturated steam was used, but became negligible when

* Proceedings Institution of Civil Engiveers, 1897-98, vol. exxxi., p. 147. 
the steam was sufficiently superheated; in fact that the reduction of this leakage was the chief cause of the economy shown when superheated steam was used. Experiments made since the date of their Paper showed that certain steam-engines driving dynamos, and which had been designed to give high economy, entirely failed to do so when using saturated steam, on account of the heavy leakages past the piston-valves, and that this leakage had been so reduced by superheating the steam that the consumption had been reduced in the proportion of 100 to 45 , a reduction which could not be explained thermodynamically.

He thought that Mr. MoLaren was aware of this two jears ago from remarks which he had made to the writer in conversation which took place at Newcastle; and since that time he himself had found that by carefully considered superheating he had been able to obtain economies from ordinary engines, which had enabled him to obtain such economy in electric light and power stationg that in two important cases he had reduced the works costs to figures far below any of those given in Mr. McLaren's Tables for condensing stations; for instance, the whole of the power at his own works had been for some years past supplied by a separate power station. In this case he had reduced the fuel in pounds and pence per unit delivered into the works from $5 \mathrm{lbs}$. and $0.42 d$. in 1900 to $3.8 \mathrm{lbs}$. and $0.32 d$. in 1903. It would have added greatly to the value of the Paper if the author had attempted to do what he did in former cost-papers addressed to the Institution of Electrical Engineers, namely, attempted in each case to find the actual weight of coal used per unit as well as its cost in pence.

Although they were greatly indebted to Mr. McLaren for bringing such interesting facts together, he was sorry to say that he could not agree with some of the conclusions the author had arrived at. For instance, on page 520, under the heading of boiler-house plant, he stated that engine-builders found much less difficulty in getting their guarantee figures from the honest steam of the Lancashire or other cylindrical boiler than they did from the high pressure "Scoteh mist" given off by the now fashionable box of water-pipes. He considered such a statement to be not only erroneous but mirchievous, 
(Lt.-Colonel R. E. Crompton, C.B.)

and it was entirely contrary to his own experience. It was no doubt made in good faith, but was in no way based on the results of average practice. No donbt Mr. McLaren and any other enginebuilder putting down plant and taking his supply of saturated steam from now and clean Lancashire boilers would find it somewhat easier under those conditions to get sufficiontly dry steam, than he would by taking the supply from equally new water-tube boilers; but watertube boilers were now almost invariably fitted with superheaters, and with reasonable management it was found in practice that, under varying conditions of load, it was far easier to obtain regularly superheated steam from these latter boilers than from Lancashire boilers with the separate superheater usually employed with them. At any rate the writer's best results, which were certainly as high as any that had ever been obtained in practice, had been obtained with water-tube boilers using chain-grate stokers, but, and here followed a condition of the greatest importance, the setting of these boilers must be as carefully considered as the design of the boilers themselves. It was only of late years that Mr. Miller bad shown at the Wood Lane joint station of the Kensington and Notting Hill Companies, that the space allowed in the combustion chambers of this class of boiler had been altogether too small when bituminous or smoky coals were used. If this matter was attended to, not only was the production of steam from this kind of boiler quite as regular and as free from moisture as from the Lancashire boilers, but the superheating could be carried on in the same flues with extreme regularity.

Another point in which he criticised the Paper was that littlo notice was taken of the question of lubrication. Whenever condensation was used, it was most desirable that the quantity of oily matter which passed into the return feed-water should be minimised, and one of the problems of the modern station engineer was to effect this. Of late years it had been found that the sight-feed lubrication was unsatisfactory. Far more satisfactory lubrication had been obtained by positive lubricators, in which either the oil or a paste of oil and graphite was forced into the steam-pipe with perfect regularity. Callendar and Nicolson drew 
aftention to the important part that lubrication played in the steam-tightness of sliding surfaces. His own experience agreed with theirs. He found that if they desired to obtain satisfactory and sufficient lubrication, so as to minimise the amount of greasy matter that went forward with the steam and still kept the sliding surfaces in the most steam-tight and perfect condition, they did so best by keeping the steam dry. All water passing forward with the steam rapidly removed the lubrication from the sliding surfaces, bat, on the other hand, he found that with supply at the higher temperature involved with regular superheat even up to a total temperature of $800^{\circ} \mathrm{F}$., on account of the facts just mentioned, surfaces could be kept in better order with a small use of oily matter than was possible with engines using saturated steam said to be sufficiently dry for econumy.

Mr. Charlis Day wrote that from the various Tables given by Mr. MoLaren it would appear that no advantage was to be derived from the adoption of condeusing at electric-lighting stations; such a conclusion was, however, quite contrary to. facts, as condensing plant properly applied was of very material benefit even in a generating station where current was supplied for lighting only, and this fact was best appreciated when the condensing plant at a station was for any reason stopped for a time.

It was to be noted that the figures from which Mr. McLaren made his deductions in regard to oondensing might equally be used to show whether water-tube boilers gave more economical results than Lancashire boilers: whether slow-speed engines ware more economical than high-speed, or for almost any comparison in regard to the equipment of electrical stations that one might wish to make. Conclusions based on figures covering such a wide area, and including so many variables, proved nothing beyond the trath of the old saying that "Statistics can prove anything."

Apart from the question of economy in coal, the Tables appeared to show that the works cost, exclusive of coal, was lower in stations which had condensing plant in addition to their engines, than it was in stations which had not got the additional condensing plant; 
(Mr. Charles Day.)

thus, in the Metropolitan non-condensing lighting stations the total works cost was $1 \cdot 632 d$. per unit, and the fuel cost $1.007 d$. per unit, the difference being $0.625 d$. per unit. Turning to those Metropolitan stations which were partly condensing, one found that instead of the difference being $0.625 d$. it was $0.604 d$. Again, in Provincial lighting and tramway non-condensing stations the works cost, exclusive of coal, was $0.56 d$. per unit, whereas the corresponding cost in the partly condensing stations was $0.338 d$, and in the condensing stations $0 \cdot 405 d$.; thus, the works cost, exclusive of coal, in the non-condensing lighting and tramway stations was about 70 per cent. higher than in those stations which had some condensing plant also.

Excluding coal, the works cost included oil, waste, water, stores, wages of workmen, repairs and maintenance. The most enthusiastic advocate of condensing plant would, he thought, hardly be prepared to say that the addition of condensing plant would reduce any of these items except water. Generally speaking, therefore, the Tables showed that condensing plant, instead of reducing fuel cost, reduced the other items forming the works cost ; this deduction was, however, so absurd that he believed the sounder conclusion was that the Tables proved nothing.

Mr. McLaren instanced (page 516) a case of 11 inches vacuum being recorded in the cylinder, whilst 23 inches were recorded in tho condenser; this case only showed that the pipes between the cylinder, or the ports into the oylinder, or both, were absurdly small, or that there was a distinct air-leakage near the cylinder. If independent condensing plant was placed within a reasonable distance of the engine with which it was connected, there was no difficulty whatever in getting a vacuum at the cylinder outlet within $\frac{1}{2}$ inch of the racuum in the condenser. The author instanced a case where the steam used by a separate condensing plant was excessive. There was no reason why such a state of affuirs should exist any more than that the steam used by the main engines should be excessive. Each could be kept satisfactory by proper attention, as had been shown in another part of the Paper.

It was stated (page 517) that when condensers were attached direct to engines the steam required by the auxiliary machinery was 
altogether saved. That statement was clearly wrong, as in either case the same amount of work had to be done in driving the pumps; the only difference in steam consumption was that arising from the possibility of the larger engine using less steam per H.P. than the smaller condensing engine. On the other hand, when an engine was but lightly loaded, the independent condensing plant might be run at half-speed, in which case the steam consumption would be less than if the pumps were driven direct from the engine. Several disadvantages were brought in when pumps were coupled to the main engine. First, the design of the main engine usually required modification to permit of direct conuected pumps. Second, it was generally now preferred to run engines driving dynamos at higher speeds than it was desirable to run air-pumps. Consequently if the pumps were so connected, they themselves were not so satisfactory as if worked independently and at lower speed; or the speed of the main engine had to be reduced to suit the pumps, with consequent increase in its cast, and in that of the dynamo. In designing engines, vibration troubles required careful consideration, and these troubles were very likely to be increased if the pumps were connected to the engine direct.

The author's suggestions as to the various ways in which improvements in economy could be obtained were excellent, but in almost every instance they applied with equal force to stations which had condensing plant as to those which had not. Mr. MeLaren stated (page 518) that 25 per cent, might be taken as the saving in fuel due to condensing, other conditions being equal. The writer's own experience quite confirmed this statement, but he would modify it by saying that, after taking into account the capital invested in condensing plant, and the slight increase of maintenance and works costs, excepting fuel and water, the safe estimate of the net gain obtained by the adoption of condensing was 20 per cent.

Mr. McLarex wrote, in further reply to those who took part in the discussion, and to the written communications since received, that it was evident from Mr. Druitt Halpin's remarks (page 558), that there was a manifest saving in fuel, by simply passing the foedwater through the storage vessel before it reached the boiler proper, 
(Mr. McLaren.)

quite apart from the advantage derived from storage. Trapping the dirt and lime at the storage ressel instead of allowing it to pass into the boiler was also a great advantage, especially where water-tube boilers were used.

Mr. George Wilkinson stated (page 546) that the losses in the mains, between the station and the consumer, might be as much as 30 to 33 per cent. where alternating current was employed. This was a very important point to be borne in mind when settling the system of supply from a central station.

Mr. Charles Forgan remarked (page 547) that if well-equipped condensing stations had been compared with the non-condensing, the former would have shown up better, which was no doubt true, but any result could have been obtained had stations been selected in each class. As already stated, the Paper was based on the broad fact that all the stations throughout the country were taken. It was impossible to compare station with station, unless the price and calorific value of the fuels used in the various stations were known, but by taking all stations, using fuel at all prices, it was possible to compare one class with another in a fair manner.

Mr. J. D. Bailie considered (page 548) that the author was rather unfair to the engineers of condensing stations. This was disclaimed by the author, who was personally a strong advocate of condensing stations, and in making suggestions where the efficiency of these stations could be improved, he did so in the most friendly spirit. The figures given (page 516), namely 23 inches at the condenser and 11 inches at the engine, were certainly the worst the author had found, and were not intended to be taken as normal conditions in condensing stations. Respecting Mr. Bailie's remarks concerning steam used by anxiliary condenser engines not being all saved, the author would call his attention to the whole of the paragraph (page 517) from which he quoted. It read that the author's firm usually guaranteed $7 \frac{1}{2}$ per cent. less steam per kw.-hour if the engines had their own condensers and drove their own pumps. The $7 \frac{1}{2}$ per cent. was the net saving in the main engines, after deducting the power required to drive the pumps. It was found that the better vacuum obtained at the engine cylinders more than 
compensated for the power required to drive the pumps. It was under these circumstances that the whole of the steam that would have beon used by the auriliaries was saved.

It was the practice in this country for engineers who made engines with separate condensers not to include the steam used by the condenser engine in their guarantee for the main engines. The author pointed out that this steam ought to be included in that need by the main engines.

Mr. Bailie also considered that the steam turbines had been treated in a disparaging manner, and objected to Newcastle District Station being compared with that of South Shields. The author admitted that there were objections to comparing individual stations, unless the whole of the conditions and cost of fuel, ete. were known. He had therefore got out the average cost per unit generated in the four turbine stations, and found that it was 5 to 6 per cent. higher than the average in the non-condensing steam-engine stations, both for coal and works costs. Great steam economy was claimed for turbines when tested individually, but the figures given in the "Electrical Times" Table of Costs did not substantiate that claim for turbine stations as a whole. Mr. Bailie added some particulars concerning Melton Mowbray and West Bromwich, to show that the costs in these stations had come down rapidly during the present jesr. This applied equally to steam-engine stations, but as the Paper only dealt with the complete year 1901 to 1902 , a period of dear coal, these additional figures could not be discussed.

Mr. Bailio in his remarks concerning superheaters said that he could not quite see that they were a disadvantage. The author agreed that superheating was a great aid to economy, and only mentioned the fact that the economy of fuel was not so marked as the economy of steam. This was especially the case where separately-fired superheaters were used.

Mr. T. L. Miller added a valuable contribution to the discussion (page 551). His remarks went to prove that the system of supply had an important berring on costs. He selected fourteen low-tension stations, and showed that the loss between the switch-board and the consumer was 13.4 per cent. and that in the high-tension stations it 
(Mr. McLaren.)

was as much as $32 \cdot 7$ per cent. The latter figures closely agreed with Mr. Wilkinson's estimate of the loss in high-tension alternating systems. Had Mr. Miller taken the whole of the stations in the country as a basis, his figures would have been more valuable, though perhaps less startling. In dealing with the coal costs of the lighting stations using low tension, he found that the corl costs in the condensing and non-condensing stations were practically identical, whereas the condensing stations ought to have shown a large saving. In the high-tension stations in London, he showed the condensing stations to come out better than the non-condensing, but to obtain this result, he omitted the Metropolitan Companies' Stations from the condensing list. They generated 11 million units out of a total of 41 millions, and their cost per unit was about 50 per cent. higher than the remaining stations which he took; whereas on the non-condensing side, he omitted Shoreditch, which only generated $2 \frac{1}{4}$ million units, at about the same cost per unit. Had be included all stations, the non-condensing side would have certainly come out the best. In the Country Lighting Stations there were only seven working non-condensing, and using the hightension system of supply. From these he selected six, the serenth (Wimbledon) having a destructor. The six selected stations were Aberystwyth, Chelmsford, Dublin, Exeter, Morley, and Watford. The total output of these stations was under $1 \frac{1}{2}$ million units, out of a total of orer 75 millions genorated in the Country Ligliting Stations. Dublin accounted for over 42 per cent. of the total units generated in the six stations, at a cost fur coal of $1 \cdot 85 d$. per unit. which was abnormally high for a large station. The cost of coal in the other five stations was also high. It would thereforo appear that hightension non-condeusing stations were not economical. Mr. Miller somehow managed to divide the whole of the current generated, as coming from separate high- or low-tension stations, but about 20 per cent. of the whole came from stations using both systems of supply. It would be interesting to know how he ascertained the amount of current supplied through each system at these stations. On the whole, Mr. Miller's figures went to prove that the system of supply had a very important bearing on the cost per unit of electricity supplied to the consumer. 
Mr. Mark Robinson mentioned (page 558) that Edinburgh and Kensington, both cconomical stations, had three-crank compound engines. The author ventured to assert that had these stations been fitted with three-crank engines, having only three cylinders, side by side (triple-expansion engines), they would have come out still better in economy. A 300-H.P. three-crank tandem compound engine was simply three engines of 100 H.P. each, and therefore had a larger proportion of loss due to clearance, initial condensation, and other losses inherent in small engines. The great economy in the stations mentioned by Mr. Robinson was no doubt largely duo to good and careful management. The author would remind him that quite number of firms made, or had made, three-crank tandem compounds.

Mr. E. G. Constanline wrote (page 564) giving some particulars concerning water-tube boilers, which he ssid might come as a surprise to the anthor, but, strange to say, he gave no figures to disprove the only thing the author inferred, namely, that the quality or dryness of the steam given off by the water-tube boiler was not so good as that given off by the cylindrical bciler. To an engine builder who guaranteed the amount of saturated steam his engine would use, the quality of the sterm was of vital importance; 10 lbs. of "Scotch Mist" per lb. of fuel might in the long run be cherper to the station engineer than $8 \mathrm{lbs}$. of honest steam, but nnder the circumstances already mentioned, the "Scotch Mist" penalised the engine builder, yet added to the apparent evaporation of the; boiler, a point to be borne in mind by the station ongineer when making engine and boiler tests. Mr. Constantine's remarks and figures were certainly interesting, but they did not bear on anything said by the author. When superheaters were added to water-tube boilers, the steam of course was dry; and good economy was obtained from its use. Mr. Constantine did not state how many of the water-tube boilers mentioned by him were fitted with superheaters. The cost of cleaning and scaling a water-tube boiler, as compared with a Lanceshire, was an important item; repairs and upkeep had also to be considered; those came in the works costs, and were important items to the station ongineer. As 
(Mr. MeLaren.)

Mr. Constantine had shown, works costs were in some cases lower where water-tube boilers were in use, and no doubt many cases could be cited where high works-costs and water-tube boilers went together.

Lt.-Colonel Crompton considered (page 567) the "Scotch Mist" statement not only erroneous, but mischievous; then he proceeded to state that it might be easier to get drier steam from a new cylindrical than a new water-tube boiler, but a water-tube boiler fitted with a superheater he considered gave good dry steam. With this statement most people would agree. The author was dealing with the quality of the steam given off by the boiler, not by the superheater.

Mr. Charles Day wrote (page 569) that he considered the conclusions drawn from the figures given in the Tables quite contrary to facts. The author would remind him that the figures given were actual facts; conclusions drawn from them might be influenced by what Mr. Day termed variables, and they might be drawn on too wide an area for comparisons to be made between individual stations. This was the very reason that induced the author to include the whole of the stations throughont the country. A wide base demanded a proportionate structure; there was no getting away from the fact that the average cost per unit of electricity generated in the whole of the non-condensing stations was 16 per cent. less than that generated in the whole of the condensing stations throughout the country. His remarks on condensers and bad vacuum \&c., had already been answered by the author in his reply to other contributors to the discussion. Mr. Day's remarks on works costs exclusive of coal were ingenious but specious. If he took the wide base, he must also take the whole structure, not select a portion of it.

In conclusion, the author desired to thank all those who had taken part in the discussion. Many points had turned up well worthy of consideration by those interested in the Economy of Fuel in Electric Generating Stations. 
FUEL ECONOMY IN GENERATING STATIONS. Pl. 14.

(Mr. Druitt Halpin's remarks.)

Fig. I. Thermal Storage of Feed Whaler for I'arying Loads.

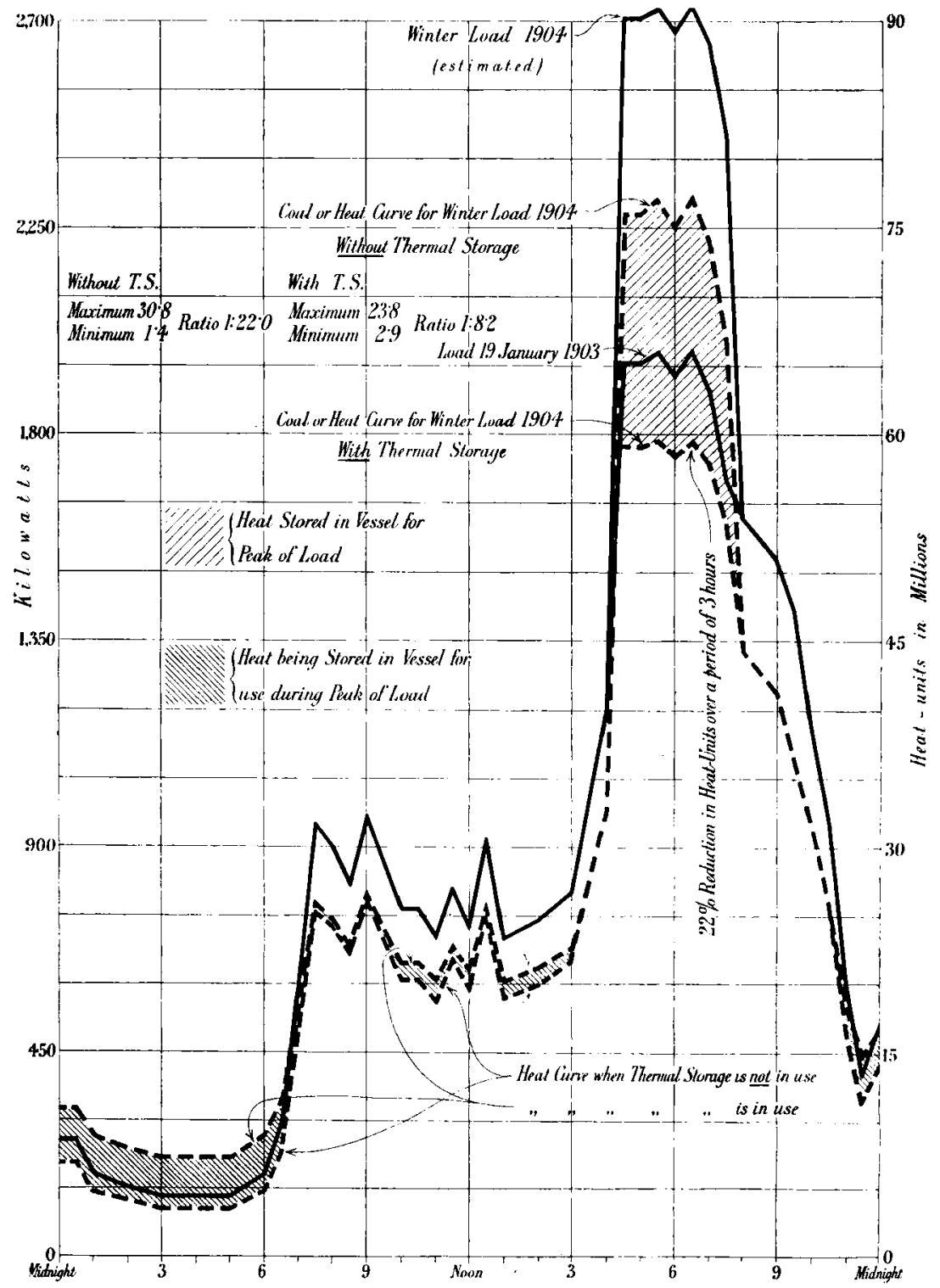


Fig. 2. Arangement applicd to a Water Tube Boiler.

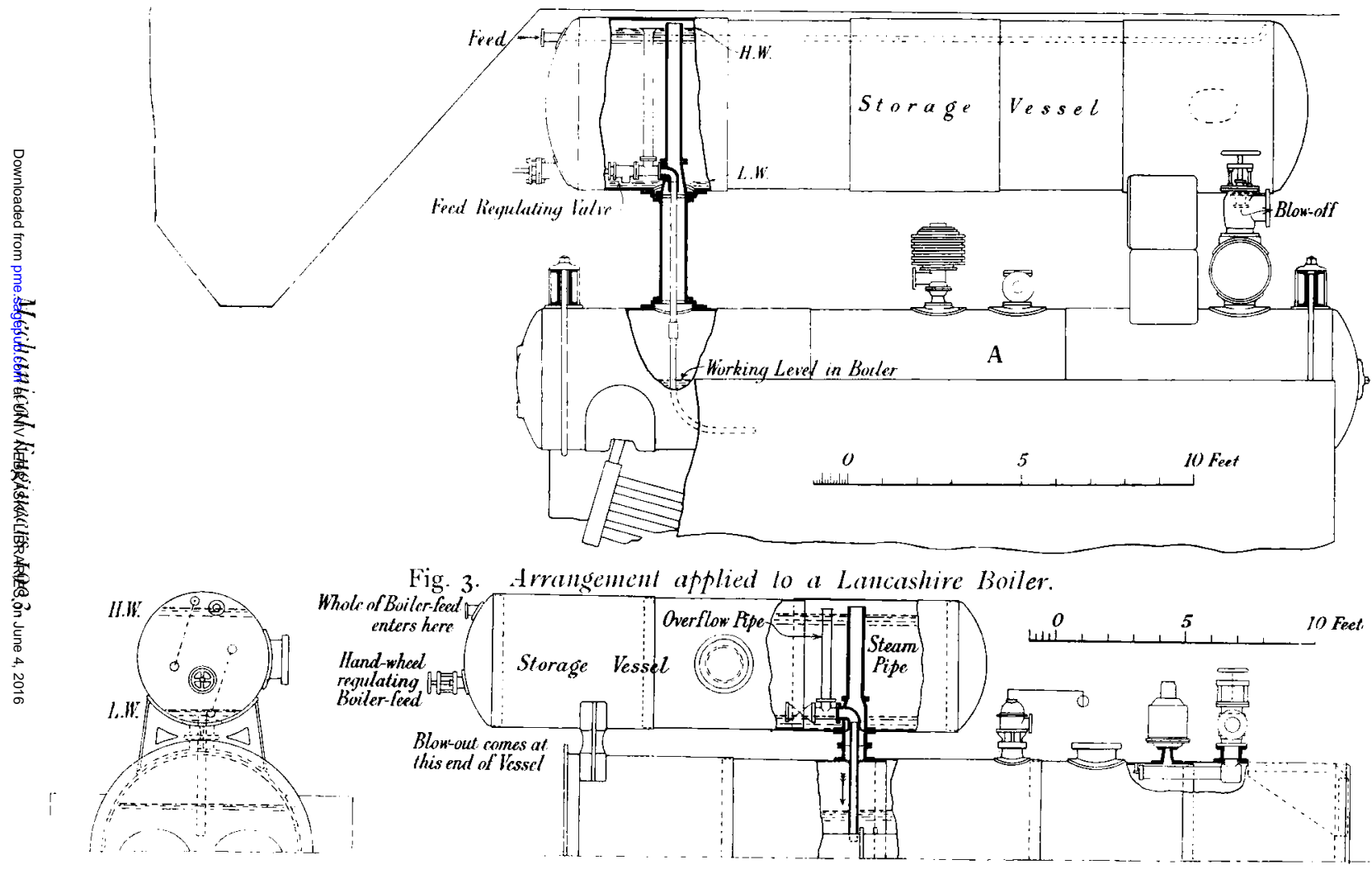

